PALEO

Revue d'archéologie préhistorique

19 | 2007

Spécial table ronde ( 1 ère partie) : Le Gravettien : entités régionales d'une paléoculture européenne, Les Eyzies, juillet 2004

\title{
l'émergence du Paléolithique supérieur en Europe : mutations culturelles et rythmes d'évolution
}

The emergence of the Upper Paleolithic in Europe: cultural mutations and

rhythms of evolution

Nicolas Teyssandier

\section{(2) OpenEdition}

Édition électronique

URL : https://journals.openedition.org/paleo/702

DOI : $10.4000 /$ paleo.702

ISSN : 2101-0420

Éditeur

SAMRA

Édition imprimée

Date de publication : 30 décembre 2007

Pagination : 367-389

ISSN : 1145-3370

\section{Référence électronique}

Nicolas Teyssandier, « l'émergence du Paléolithique supérieur en Europe : mutations culturelles et rythmes d'évolution », PALEO [En ligne], 19 | 2007, mis en ligne le 23 avril 2009, consulté le 25 juin 2021. URL : http://journals.openedition.org/paleo/702 ; DOI : https://doi.org/10.4000/paleo.702

\section{(c) (†) $\odot$}

PALEO est mis à disposition selon les termes de la licence Creative Commons Attribution - Pas d'Utilisation Commerciale - Pas de Modification 4.0 International. 


\title{
L'ÉMERGENCE DU PALÉOLITHIQUE SUPÉRIEUR EN EUROPE : MUTATIONS CULTURELLES ET RYTHMES D'ÉVOLUTION
}

Nicolas TEYSSANDIER ${ }^{(1)}$

\begin{abstract}
Résumé : La mise en place du Paléolithique supérieur est traditionnellement envisagée sous l'angle de la rupture. De ce point de vue, l'Aurignacien est interprété comme " LA " culture qui consacrerait la généralisation de nombreuses innovations et représenterait l'instrument de la conquête européenne de l'Homme moderne et du remplacement des populations néandertaliennes. Cet article fait le point sur la définition actuelle des débuts de l'Aurignacien européen et essaie de les restituer dans un processus évolutif. L'un des moteurs de l'évolution technologique des groupes humains entre 45000 et $30000 \mathrm{BP}$ pourrait correspondre à la recherche de solutions techniques pour armer des projectiles. De ce fait, une plus grande profondeur temporelle et une relative arythmie dans le développement des caractères constitutifs du Paléolithique supérieur sont mises en avant. A ce titre, la distinction chronologique et technologique d'au moins deux grandes traditions - le Protoaurignacien et l'Aurignacien ancien - est évoquée, conduisant à modifier substantiellement les schémas d'évolution classiques du Paléolithique moyen au Paléolithique supérieur.
\end{abstract}

Mots-clés : Paléolithique supérieur, Protoaurignacien, Aurignacien ancien, industries de transition, Homme moderne, projectile, pointe lithique, lamelle, technologie lithique, leptolithisation, processus d'évolution.

Key-words : Upper Paleolithic, Protoaurignacian, Early Aurignacian, Middle-to-Upper Paleolithic transition, Modern Humans, projectile, lithic point, bladelet, lithic technology, leptolithisation, evolutionnary process.

\begin{abstract}
Abridged english version
THE EMERGENCE OF THE UPPER PALEOLITHIC IN EUROPE : CULTURAL MUTATIONS AND RHYTHMS OF EVOLUTION.

The notion of a rupture dominates reflections on the emergence of the Upper Paleolithic in Europe. From this perspective, the Aurignacian is interpreted as the culture that would realize the systematization of numerous innovations and become the instrument of victory of Modern Humans over Neandertal populations. In this way, the Aurignacian is perceived as the cultural entity whose different constitutive elements - blade and bladelet production, systematization of organic tool production, personal ornaments, portable and parietal art - generically define the Upper Paleolithic and distinguish it from the Middle Paleolithic. Based on these technical and symbolic innovations, the Aurignacian is represented as a homogeneous and conquering pan-European culture whose origin and expansion are forcibly linked to the unique trajectory of modern humans. The formalization of explicit models associating the development of the Aurignacian with the emergence of modern humans in Europe has thus gradually blurred the variability of this techno-complex, leaving only a skeletal image composed of carinated end-scrapers, Aurignacian blades and split-based points. Our objective is not to minimize the magnitude of changes that occurred with the emergence of the Aurignacian, but rather to present elements in support of the hypothesis that these changes were the result of an evolutionnary process whose different stages remain to be defined. To put this discussion into context, we shall first review current definitions of the early stages of the European Aurignacian.
\end{abstract}

\section{The beginnings of the Aurignacian : multiple technical and cultural expressions and/or chronological filiation?}

Several manifestations of the Aurignacian were already defined many years ago. Among the most significant and clearly expressed in the archaeological record are the Protoaurignacian and the Early Aurignacian. 


\section{The Protoaurignacian : a Mediterranean or European reality ?}

Since the 1960's and the work by G. Laplace, the industries of an initial Upper Paleolithic characterized by the presence of long retouched bladelets were isolated under the denomination Protoaurignacian. They were gradually defined over the entire west-Mediterranean perimeter. However, for several reasons, these industries were gradually erased from the broad syntheses, or at most appeared at the same level as the more classic assemblages, all grouped under the generic Aurignacian denomination. Recent researches have allowed us to enlarge their geographical repartition and to better define their chronological position. In central Europe, no sites have yielded evidence of a Protoaurignacian/Early Aurignacian stratigraphic succession. Until recently, the only site considered to contain a Protoaurignacian component was the open-air occupation of Krems-Hundssteig in Lower Austria. Unfortunately, its rich lithic assemblage was not found in stratigraphic context. The lithic material is characterized by a large number of retouched bladelets including more than 1,500 Dufour bladelets with altemate retouch associated with unipolar bladelet cores of pyramidal morphology (fig. 1). The complexity of the interpretation of the Krems asssemblage is exacerbated by the presence of wide-fronted carinated cores (fig. 1, $n^{\circ}$ ) and robust blades with lateral retouch (fig. 1, $n^{\circ} 7-8$ ) that are more typical of the Early Aurignacian. In central Europe, assemablages with long retouched bladelets that could be integrated into the Protoaurignacian tradition are still rare and partially known. Nevertheless, the open-air site of Tincova (Romania) is a good candidate for integration within this initial stage of the Aurignacian. The dominant intention of the lithic productions was to fabricate elongated bladelets with a rectilinear profile. As at Krems, there is long bladelets pointed by direct bilateral retouch (i.e. Krems point, fig. 2, $n^{\circ} 1-3$ ) and Dufour type bladelets with alternate retouch (fig. $2, n^{\circ} 5,7$ ). This assemblage does not seem to be geographically isolated since in northwestern Bulgaria, layer VII of Kozarnika cave dated around 39 000-36 000 BP show close similarities (fig. 2, $\mathrm{n}^{\circ}$ 8-14).

The escalating number of industries with long retouched bladelets in the initial stage of the Upper Paleolithic requires us to substantially modify our evolutionary schemes. It now appears that the geographic distribution of these industries extends largely beyond the Mediterranean coast into diverse ecosystems, attesting to the success of the technical ideas they conveyed. Moreover, they are stratrigraphically and culturally distinct from the "classic" Early Aurignacian. We shall now review the definition of the Early Aurignacian.

\section{The Early Aurignacian (I) and the example of the Swabian Jura}

The Early Aurignacian is the best known episode of the Aurignacian. Recent analyses of lithic industries of key sequences have led to a clarification of the unity of the Early Aurignacian in southwest France. A strong technical identity is observed in lithic productions, which are oriented toward the fabrication of blades and bladelets through distinct core reduction methods (fig. 4). Originally defined in southwestern France, the Early Aurignacian is also clearly recognized in the Swabian Jura region of Southern Germany where it has been convincingly defined at Geissenklösterle cave (fig. 5). Elsewhere in central Europe, the Early Aurignacian appears to be more diffuse. Clear Early Aurignacian signatures, including split-based points, exist as far as the Near-East but they seem to post-date European ones. Moreover, Early Aurignacian sites in an intermediate geographical position between Western Central Europe and the Middle East remain rare.

\section{The origins myth}

With the initial unity of the Aurignacian now being largely questioned, its origins have become increasingly complex and obscure. In this context, the Bachokirian, long interpreted as a formative "facies" of the Aurignacian in Europe, is important. Recent analyses of the eponymous industry of level 11 of Bacho Kiro (Bulgaria) reveal characteristics that are clearly distinct from the common Aurignacian norms (fig. 6). The assemblage is more directly comparable to that of lithic industries situated at the boundary between the Middle and the Upper Paleolithic (fig. 7). They show the beginnings of an increased blade production (leptolithisation) associated with the development of technical solutions for the production of generally elongated, mostly convergent blanks or points. The Bachokirian may thus be part of the vast mosaic characterized as early as 50 000-45 $000 \mathrm{BP}$ by a multiplication of blade and point productions employing a mixture of Levallois and more classic Upper Paleolithic method and using only direct hard hammer percussion.

\section{Are weapons a common denominator?}

One common tendency can be distinguished among the nebula of so-called Middle-to-Upper paleolithic transitional industries: the production of relatively light and convergent blanks. This "point" phenomenon seems to be anchored in local, Levallois type substrata. One of the motivating factors of change in lithic productions at the dawn of the Upper Paleolithic was the role played by projectile elements that are commonly fabricated on light and elongated lithic blanks. This phenomenon of "leptolithisation", though is clearly evident form the beginning of the Aurignacian, is not completely new in the evolutionnary trajectory of the Eurasian Paleolithic and its roots may exist in the preceding mosaic of "transitional" point complexes. Therefore, a common preoccupation, in this case the search for technical solutions for arming projectiles, could explain the apparent community of these distinct industrial complexes (Emiran, Bachokirian, Bohunician...). It is not until later that this tendency would become concrete and lead to a relative homogenization of technical traditions across Europe (Protoaurignacian) and the Near East (Early Ahmarian). This apparent unification of technical traditions could be attributed to the remarkable success of bladelet productions from both a techno-functional and socio-economic perspectives. Around $35000 \mathrm{BP}$, a large part of Europe shifted to the Aurignacian system and clear regional variations emerged, particularly in the domain of display objects such as personal omaments (fig. 8). Therefore, the long evolution that represents the so-called Middle-to-Upper Paleolithic transition could correspond to a modification of social interactions between groups, leading to the large-scale diffusion of structuring ideas or technical solutions.

\section{Conclusion}

Despite numerous obscurities still surrounding the formulation of these hypotheses, several consensuses remain to be established:

- The first concerns to the anterior position of industries with long retouched bladelets (Early Ahmarian, Protoaurignacian) relative to those with carinated end-scrapers and split-based points (Early Aurignacian).

- The secund concerns the geographic extension of the "long retouched bladelet" phenomenon from the zone of its initial identification in the Westem Mediterranean area to Austria, Romania and as far as the Balkans. Long thought to be restricted to Westem Europe, it is now considered to be a pan-European entity. In contrast, and given that it is posterior to the Protoaurignacian, the classic Early Aurignacian appears to be a European phenomenon arising from changes that had already developed in other technical traditions.

- Finally, our perception of sudden and brutal innovations at the very beginning of the Aurignacian is now challenged in favour of more gradual appearance of the constitutive elements of the emerging Upper Paleolithic. 
La notion de rupture jalonne les réflexions sur l'émergence du Paléolithique supérieur. Comment ne pas voir en effet au travers de ces parures, peintures, blocs sculptés, formes animales dégagées de l'ivoire ou pointes de sagaie en matières dures animales, la trace d'une mutation significative et quelque peu abrupte dans la longue histoire de l'évolution humaine. En Europe, l'apparition "soudaine " de ces manifestations a rapidement été associée à celle d'un nouveau type humain (Breuil 1913), Homo sapiens sapiens. Depuis longtemps (voir p. ex. Sonneville-Bordes 1960, p.150) et plus encore depuis la découverte de restes humains néandertaliens en contexte Châtelperronien à Saint-Césaire (Lévêque et Vandermeersch 1980) et les refontes paradigmatiques qu'elle imposa, l'apparition de l'Homme moderne en Europe est perçue sous les traits de l'Aurignacien ancien, " culture " que l'on se représente depuis longtemps déjà comme homogène par la remarquable répétition de caractères communs sur une vaste aire géographique (voir p. ex. Sonneville-Bordes 1960 ; Mellars 1989, 2004), allant des rivages atlantiques aux monts du Zagros. L'emblème de l'unité aurignacienne serait notamment illustré par la systématisation des productions en matières dures animales, cristallisée autour d'une extrémité de projectile à l'emmanchement particulier, la pointe de sagaie à base fendue.

Sur un plan culturel, c'est donc l'Aurignacien qui va, aux yeux des préhistoriens, représenter l'instrument de la conquête de l'Homme moderne et du remplacement de son prédécesseur néandertalien. A ce titre, l'Aurignacien est envisagé comme "LA " culture dont les différents caractères constitutifs - débitage de lames et de lamelles, systématisation des productions en matières dures animales, parures, art mobilier et pariétal - définissent, d'un point de vue générique, le Paléolithique supérieur en le distinguant du Paléolithique moyen.

Allant de pair avec ces innovations technologiques et symboliques, la représentation de l'Aurignacien va être associée à celle d'une culture pan-, voire même supra-européenne (voir p. ex. Mellars 1989, 2004 ; Kozlowski 1993 ; Kozlowski et Otte 2000), homogène et conquérante, dont l'origine et l'expansion ne peuvent qu'être liées à la trajectoire singulière de l'Homme moderne. La formalisation de modèles explicites associant le développement de l'Aurignacien à l'émergence de l'Homme moderne en Europe (voir p.ex. Demars et Hublin 1989 ; Mellars 1989, 2004 ; Otte 1990 ; Kozlowski ibid. ; Kozlowski et Otte ibid. ; Davies 2001) va, peu à peu, gommer la variabilité de ce techno-complexe pour n'en retenir qu'une image d'Epinal, faite de grattoirs carénés, de lames aurignaciennes et de pointes de sagaie à base fendue. Pourtant, tout au long du $X X^{e}$ siècle, nombreux sont ceux, en particulier en Europe centrale et orientale ou en péninsule ibérique, qui ont décrit des situations plus polymorphes quant à la formation et au développement de l'Aurignacien (voir p.ex. Hahn 1977 ; Clark et Lindly 1990 ; Valoch 1990 ; Straus 1996, 2003 ; Cabrera Valdés et al. 2001) et défendu l'idée d'une plus grande complexité du phénomène aurignacien, perception récemment renforcée par les études technologiques des productions matérielles sur lesquelles nous nous appuyons.

À travers cette approche, nous ne chercherons pas à nier, bien au contraire, l'ampleur des changements qui vont prendre place avec l'Aurignacien mais essaierons plutôt d'étayer l'hypothèse que ceux-ci sont le fruit d'un processus évolutif dont les différentes étapes demandent à être redéfinies (voir p.ex. Sonneville-Bordes 1960). Nous nous interrogerons par conséquent sur la profondeur historique et le sens évolutif des mutations qui vont, effectivement, se concrétiser avec l'émergence puis le développement des sociétés aurignaciennes. Mais voyons auparavant comment les débuts de l'Aurignacien européen peuvent désormais être définis.

Les débuts de l'Aurignacien : pluralité des expressions techniques et culturelles et/ou filiation chronologique ?

Depuis longtemps déjà, plusieurs expressions de l'Aurignacien ont été identifiées ; parmi les plus significatives et les mieux exprimées dans la documentation a rchéologique, se côtoient un "Protoaurignacien " (Laplace 1966) et un "Aurignacien ancien " (SonnevilleBordes 1960). Ces deux grands types d'industries se différencieraient d'un point de vue chronologique, en faveur d'une plus grande ancienneté du Protoaurignacien, mais également au niveau de leur répartition géographique : un $P$ rotoaurignacien méditerranéen étant opposé à un Aurignacien ancien plus continental. Cette distinction a curieusement été " oubliée "lors de la formalisation de grandes synthèses sur le peuplement de l'Europe par les premiers hommes modernes, pour ne retenir que l'idée de la grande unité du peuplement aurignacien (voir p.ex. Mellars 1989, 2004 ; Otte 1990 ; Kozlowski 1993 ; Kozlowski et Otte 2000). Au mieux, cette dualité d'expression des premières formes de l'Aurignacien était conservée pour distinguer deux voies principales de " colonisation " qui suivraient d'abord un cheminement commun, le long du bassin inférieur du Danube, avant de se séparer, l'une remontant le cours de ce grand fleuve, tandis que l'autre bifurquerait pour rejoindre les côtes méditerranéennes (Djindjian 1993). Ce schéma implique donc des racines communes à ces groupes d'industries qui se démarqueraient, dans un second temps, au cours de leur cheminement géographique différent. Les données récentes ne semblent plus appuyer ce modèle et tendent, au contraire, à distinguer ces deux formes d'expression de l'Aurignacien et à les voir, dans certaines régions au moins, se succéder dans le temps.

\section{Le Protoaurignacien : une réalité méditerranéenne ou européenne?}

Depuis les années 1960 et les travaux de G. Laplace (1966), des industries d'un Paléolithique supérieur initial se caractérisant par la présence de grande lamelles retouchées ont été isolées sous l'appellation "Protoaurignacien ". Elles ont peu à peu été définies sur tout le pourtour ouest-méditerranéen et, plus particulièrement, au nord de l'Italie (Bartolomei et al. 1994 ; Kuhn et Stiner 1998 ; Broglio et al. 2005), dans le Sud-Est français (Bazile 1974, 2006 ; Onoratini 1986, 2004), au Pays Basque et dans les Pyrénées françaises (Laplace 1966 ; Laplace et al. 2006 ; Normand et Turq 2005 ; Normand 
2006), jusqu'en Catalogne (Maroto et al. 1996 ; Ortega Cobos et al. 2005) et dans les Cantabres (Maillo Femandez 2005). Mais, en partie parce que les travaux de G. Laplace ne trouvaient pas confirmation auprès des séquences classiques d'Aquitaine (Djindjian 2006) et qu'ils se heurtaient à la désapprobation de plusieurs chercheurs (voir p.ex. Bordes 1963 ; Sonneville-Bordes 1958, 1966), ce "Protoaurignacien " a peu à peu été gommé des nombreux travaux de synthèse, si ce n'est pour y apparaître au même niveau que des ensembles plus classiques, regroupés sous l'appellation générique d'Aurignacien. A ce titre, la variabilité des débuts de l'Aurignacien était masquée pour n'en retenir que son homogénéité pan-européenne et ses caractères jugés les plus significatifs.

Depuis, des travaux récents ont permis de mieux cemer cette variabilité, dans un grand quart sud-ouest de la France notamment, et la distinction "Protoaurignacien " / "Aurignacien ancien " a été posée sur des bases élargies, les analyses technologiques (Bon 2002 ; Bordes J.-G. 2006) venant appuyer la validité de ce découpage. Parallèlement, l'aire initiale du Protoaurignacien s'est trouvée étendue à l'Aquitaine où la reprise des travaux au Piage, dans le Lot, est par ailleurs venue confirmer le résultat des datations radiométriques et la position basale de ces industries au sein de la séquence aurignacienne (Bordes J.-G. 2002, 2006), démonstration appuyée également par la reprise des fouilles sur la séquence d'Isturitz (Normand 2006). Enfin, la prise en compte de la culture matérielle et symbolique des ensembles " protoaurigna ciens " est venue poser la question de leur stricte différenciation vis-à-vis de leurs homologues-successeurs " aurignaciens anciens" (Bon ibid. ; Teyssandier 2003, 2006).

En Europe centrale, terrain privilégié de nos enquêtes (Teyssandier 2003, 2006, 2007 ; Teyssandier et al. 2006), aucun site ne témoigne d'une succession stratigraphique "Proto/ancien ". Jusqu'à récemment, l'unique gisement recelant avec certitude une composante protoaurignacienne était la station de plein air de Krems-Hundssteig, en Basse-Autriche, connue depuis le début du XX ${ }^{\text {e }}$ siècle (Strobl et Obermaier 1909). Le richissime matériel lithique mis au jour, estimé entre 50000 et 70000 pièces, n'est pas le produit d'une fouille archéologique mais d'une collecte effectuée lors de l'exploitation des loess en vue de la construction d'une digue de protection contre les crues du Danube. Ce matériel n'a donc pas été retrouvé en contexte stratigraphique et, au vu des dessins représentant les zones de découverte, on ne peut que mettre en doute l'affirmation de J. Strobl et H. Obermaier (1909) évoquant un complexe archéologique absolument unitaire. Ce matériel se caractérise par une grande abondance des lamelles retouchées, parmi lesquelles J. Hahn a décompté plus de 1800 lamelles Dufour dont 1565 spécimens à retouche alterne (Hahn 1977). Celles-ci composent une part indubitablement " protoaurignacienne " de l'ensemble archéologique, d'autant plus qu'une étude technologique (Teyssandier 2003, 2007) a permis de montrer que leurs caractères métriques et techniques permettaient de les associer à de nombreux nucléus à lamelles (fig. 1). Ceux-ci sont fréquemment de morphologie pyramidale et présentent certaines spécificités (fig.1, $\mathrm{n}^{\circ}$
6) - morphologie triangulaire de la surface lamellaire assurée par l'extraction d'enlèvements lamellaires latéraux convergents en partie distale de la table - déjà observées dans les ensembles du Protoaurignacien d'Europe occidentale (Bon 2002). La difficulté de l'interprétation de l'ensemble de Krems-Hundssteig est également renforcée par la présence de grattoirs carénés à front large (fig. $1, n^{\circ} 9$ ) et de lames larges et robustes (fig. 1, n 7-8), pour certaines aménagées en grattoirs ou en lames à retouche latérale tendant parfois vers la lame aurignacienne ou la lame étranglée (Teyssandier 2003, 2007). Ces éléments sont habituellement plus typiques de l'Aurignacien ancien (Sonneville-Bordes 1960 ; Bon 2002 ; Bordes J.-G. 2006). A ce titre, l'absence de points de comparaisons régionaux perturbe l'interprétation de l'industrie d'Hundssteig et nous ne pouvons pour l'heure présumer si les composantes de type Protoaurignacien et Aurignacien ancien correspondent à un " syncrétisme technique " ou si, plus simplement, elles évoquent l'hétérogénéité et les pollutions subies par les niveaux archéologiques originels. Nous sommes plutôt enclins à considérer favorablement la seconde hypothèse, d'autant que des fouilles récentes montrent que le site a également été fréquenté à différents moments du Gravettien (NeugebauerMaresch, communication personnelle).

De tels ensembles à grandes lamelles retouchées sont pour I'heure rares et trop partiellement documentés en Europe centrale. Cependant, suite à la révision de nombreuses séquences européennes et au constat selon lequel le Protoaurignacien aurait une répartition géographique étendue, de nouveaux points de comparaison se doivent d'être mentionnés. J. Zilhão (2006) a évoqué l'exemple de Tincova, dans le Banat, au sud-ouest de la Roumanie. Ce site de plein air, fouillé dans les années 1950, présente apparemment un unique ensemble paléolithique contenant une industrie lithique rappelant le Protoaurignacien occidental. Un examen détaillé de la bibliographie disponible (Hahn 1977 ; Mogosanu 1983 ; Paunescu 2001) et une première mission préliminaire sur place en collaboration avec lon Cornel Baltean (Université de lasi, Roumanie) confirment cette proposition. L'intention dominante des productions de Tincova correspond à l'obtention de lamelles élancées au profil rectiligne. On note schématiquement, comme à Krems, la présence de deux populations de lamelles retouchées (fig. 2) : de grandes lamelles appointées par retouche bilatérale directe (pointes de Krems, fig. 2, $n^{\circ} 1-3$ ) et des lamelles plus courtes, de type Dufour (sous-type Dufour, fig. 2, $n^{\circ} 5,7$ ), à retouche alterne, directe sur le bord gauche et inverse sur le bord droit (Demars et Laurent, 1989). Celles-ci sont accompagnées d'un outillage relativement classique pour l'Aurignacien, fait principalement de grattoirs simples sur lames et de lames retouchées. Les grattoirs carénés sont rares et la retouche aurignacienne absente. Ces caractères font de Tincova un bon candidat pour une intégration au sein de la tradition protoaurignacienne à grandes lamelles retouchées. En outre, cet ensemble ne semble pas isolé géographiquement puisque au nordouest de la Bulgarie, les travaux de T. Tsanova (2006) ont nettement fait ressortir les caractères distinctifs de la couche VII de la grotte de Kozarnika. Nous souscrivons ainsi tout à fait à son interprétation, comparant le "Kozarnikien " au Protoaurignacien. 

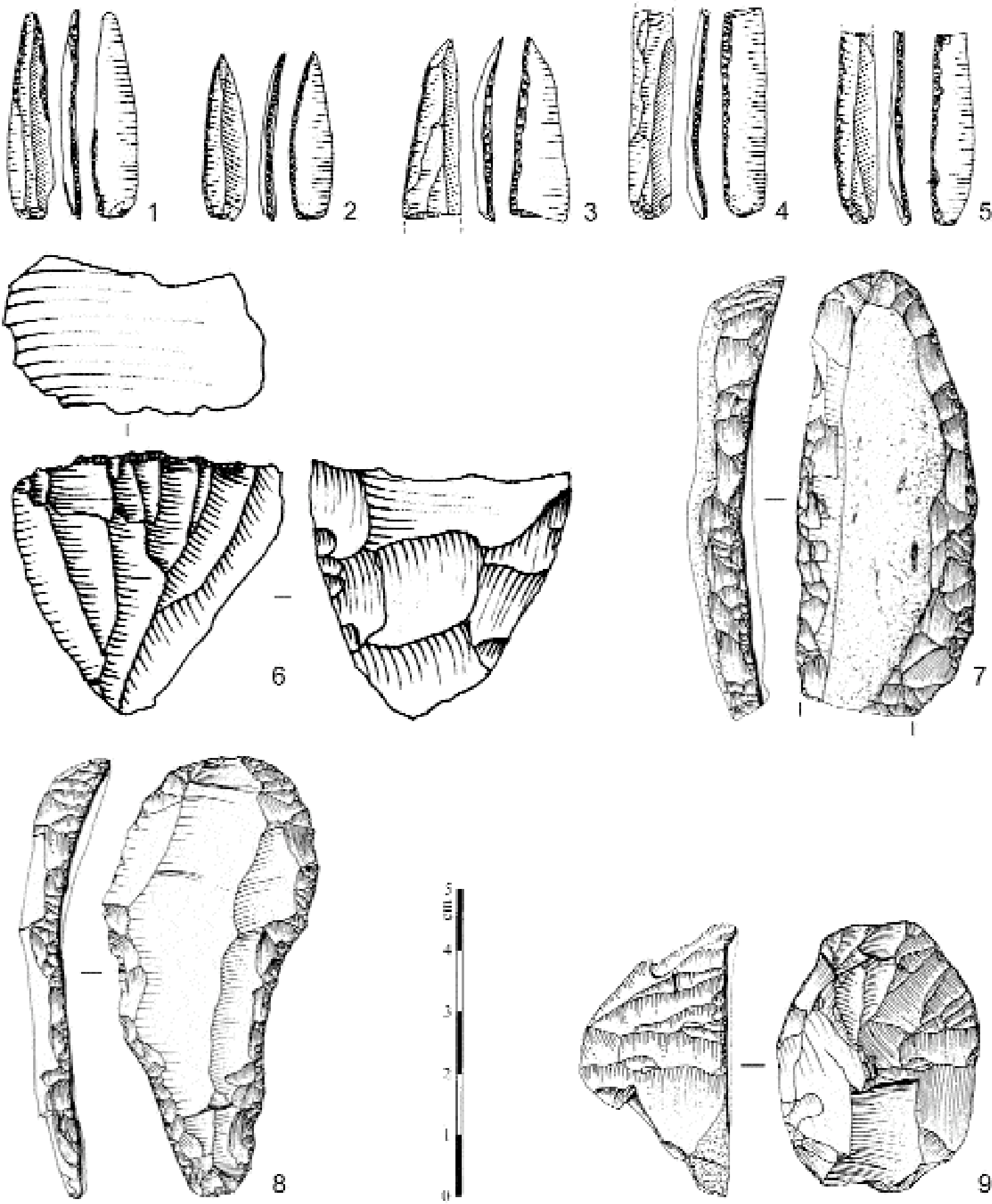

Figure 1 - Industrie lithique de Krems-Hunddsteig, Autriche (1-5 et 7-9, d'après Hahn 1977 ; 6, d'après Teyssandier 2003). 1-5: lamelles retouchées de type Dufour ; 6 : nucléus à lamelle de morphologie pyramidale ; 7-8 : grattoirs sur lames retouchées ; 9 : nucléus ("grattoir ") caréné à front large. Noter que les pièces $n^{\circ} 1$ à 6 s'intègrent parfaitement à la tradition du Protoaurignacien alors que les $n^{\circ} 7$ à 9 s'apparentent davantage aux caractères classiques de l'Aurignacien ancien.

Figure 1 - Lithic industry from Krems-Hunddsteig, Austria (1-5 and 7-9, after Hahn 1977; 6, after Teyssandier 2003). 1-5: retouched bladelets, Dufour type; 6: pyramidal bladelet core; 7-8: end-scrapers on retouched blades; 9: carinated core ("scraper") with a wide front. Note that pieces $n^{\circ} 1$ to 6 can be fully integrated in the Protoaurignacian tradition, while $n^{\circ} 7$ to 9 comespond more closely to the classic characteristics of the Early Aurignacian. 

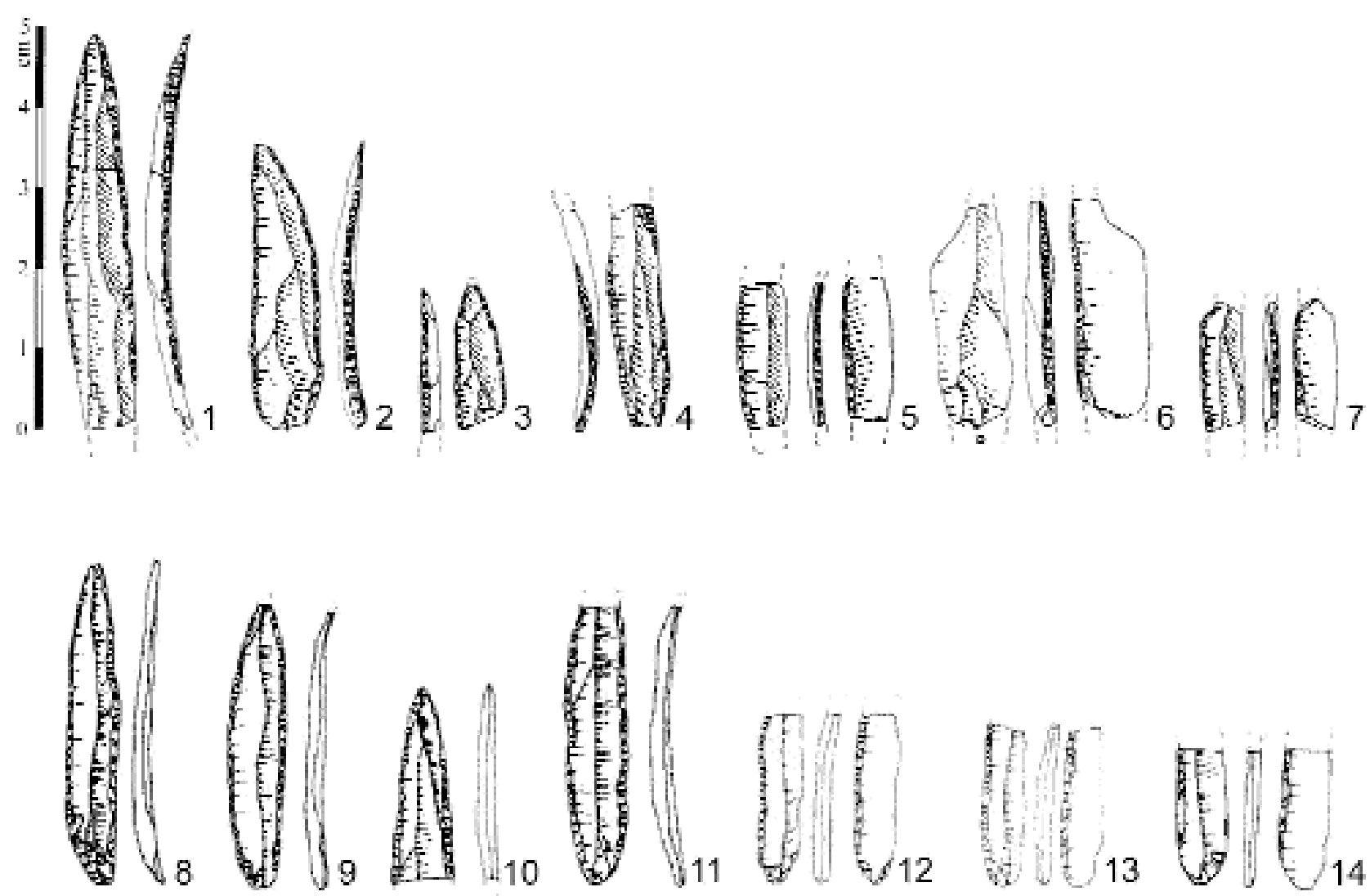

Figure 2 - Industrie lithique de Tincova, Roumanie (1-7, d'après Hahn 1977) et de la couche VII de Kozarnika, Bulgarie (8-14, d'après Tsanova 2006). 1-3 et 8-11 : lamelles appointées par retouche bilatérale directe ; 4 : lamelle à retouche bilatérale directe ; 5-7 et 12-14 : lamelle Dufour à retouche alterne.

Figure 2 - Lithic industry from Tincova, Romania (1-7, after Hahn 1977) and level VII of Kozarnika, Bulgaria (8-14, after Tsanova 2006). 1-3 and 8-11: bladelets pointed by direct bilateral retouch; 4: bladelet with direct bilateral retouch; 5-7 and 12-14: Dufour bladelet with alternate retouch.

Figure 3 - Quelques exemples d'outils en matières dures animales du Paléolithique supérieur initial. 1-2 : fragments de baguette (sagaie ?), Les Abeilles, Haute-Garonne, couche 2, Protoaurignacien (d'après Laplace et al. 2006 ; ces deux pièces sont dites en bois de cervidé dans la présente publication alors que G. Laplace les désigne comme des pièces en ivoire en 1966. F. Bon les a observé récemment et penche lui aussi pour de l'ivoire (communication personnelle) ; 3 : fragment mésio-distal de pointe de sagaie de section ovalaire, Le Piage, Lot, couche K, Protoaurignacien (d'après Mons 1981); 4 : sagaie de section circulaire en ivoire, Geissenklösterle, Allemagne, ensemble III, Aurignacien ancien (d'après Hahn 1988); 5 : baguette d'ivoire de section circulaire, Grotte du Renne à Arcy-sur-Cure, Yonne, couche VII, Protoaurignacien (d'après Julien et al. 2002). Toutes ces pièces présentent la particularité d'être de section circulaire. II pourrait s'agir là d'un caractère particulier des productions osseuses du Paléolithique supérieur initial.

Figure 3 - A few examples of tools in osseous materials from the initial Upper Paleolithic. 1-2: rod fragments (spear point?), Les Abeilles, Haute-Garonne, level 2, Protoaurignacian (after Laplace et al. 2006; in the present publication, these two pieces are said to be antler, while G. Laplace designated them as ivory in 1966. F. Bon recently examined them and also favors ivory, personal communication); 3: mesio-distal fragment of a spear point with an oval section, Le Piage, Lot, level K,

Protoaurignacian (after Mons 1981); 4: ivory spearpoint with a circular section, Geissenklösterle, Germany, AH III, Early Aurignacian (after Hahn 1988); 5: ivory rod with a circular section, Grotte du Renne at Arcy-sur-Cure, Yonne, level VII, Protoaurignacian (after Julien et al. 2002). All these pieces have a circular section. This could be particular characteristic of osseous productions in the initial Upper Paleolithic. 


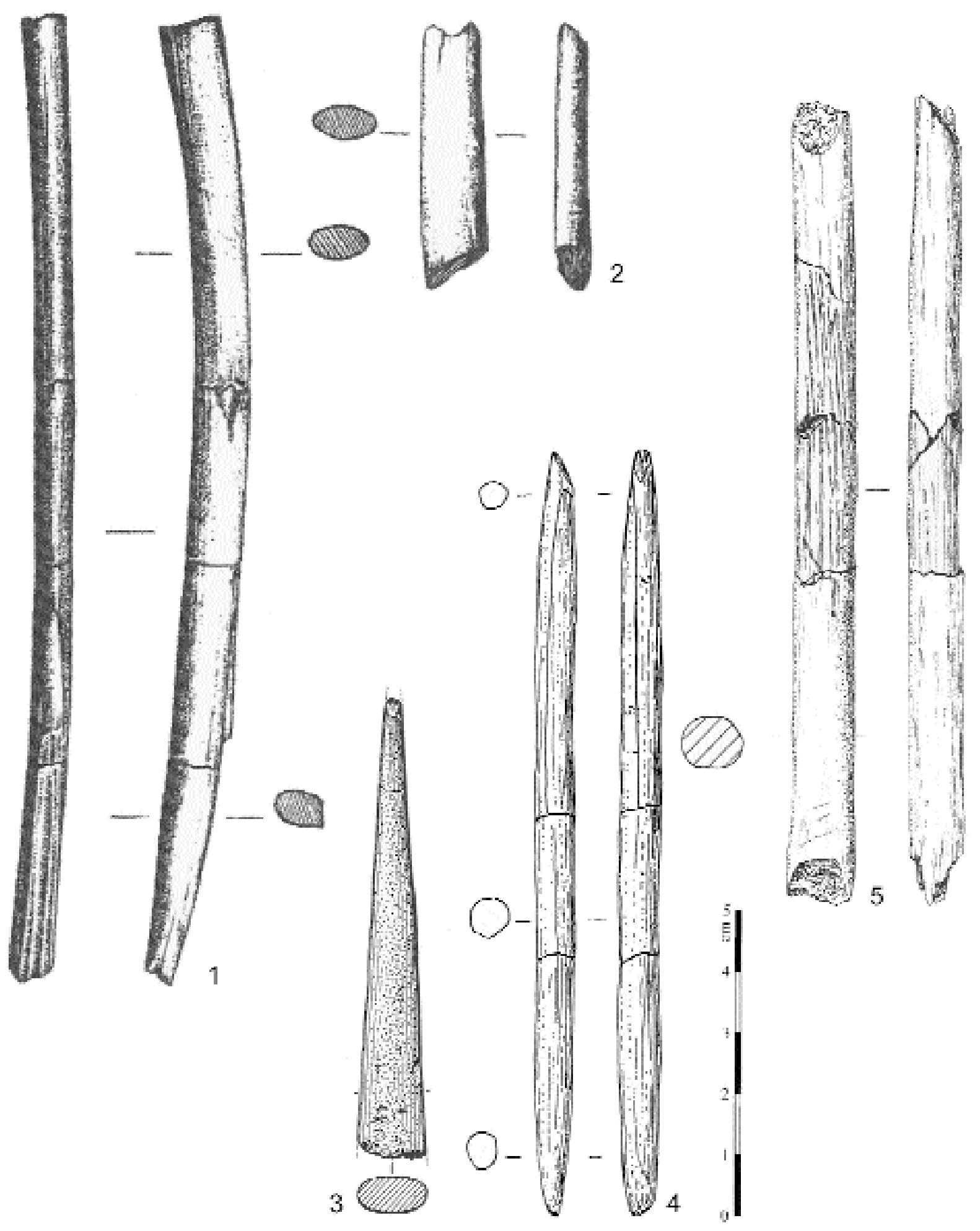


L'étude technologique montre que lames et lamelles sont obtenues dans un continuum opératoire aux dépens des mêmes nucléus et que, comme à Tincova, l'intention de produire des supports allongés, légers et rectilignes est dominante et vise la fabrication de lamelles pointues confectionnées par une retouche directe, marginale et bilatérale évoquant les pointes de Krems (fig. 2, 8-10) et de lamelles Dufour (sous-type Dufour, fig. 2, $n^{\circ} 12-14$ ) à retouche alterne. Cette couche VII constitue, à Kozarnika, la base de la séquence du Paléolithique supérieur et 3 dates ${ }^{14} \mathrm{C}$ la situent entre 39000 et 36000 BP (Guadelli et al. 2005).

En ce qui concerne les autres éléments de la culture matérielle et symbolique des groupes protoaurignaciens, les données demeurent encore totalement lacunaires pour l'Europe centrale où peu d'autres matériaux sont conservés sur les sites considérés. Pour l'Europe occidentale, les données sont plus fournies même s'il demeure délicat de les synthétiser tant les corpus sont inégaux. Tout juste peut-on constater que, sur la plupart des gisements concernés, les productions en matières dures animales semblent plus pauvres et moins diversifiées qu'elles ne le seront avec l'Aurignacien ancien (Liolios 2006). Toutefois, compte-tenu de la localisation géographique de nombreux gisements protoaurignaciens à proximité du pourtour méditerranéen, il convient de rester prudent quant à une éventuelle indigence des productions osseuses au début de l'Aurignacien. On sait en effet que, dans ces régions, les outillages en matières dures animales demeurent généralement moins développés qu'ils ne le sont en Europe continentale et ce, tout au long du Paléolithique supérieur. A titre d'exemple, les travaux de L. Mons (1981) sur la série de la couche $\mathrm{K}$ du Piage, désormais attribuée au Protoaurignacien (Bordes 2002), faisaient état de la présence de pointes et de poinçons de plusieurs types. Celle-ci rendait compte également de l'absence totale de pointes à base fendue et de l'aspect particulier de plusieurs fragments de pointe de section circulaire (fig. $3, n^{\circ} 3$ ). Ce pourrait être là un caractère particulier de ces productions osseuses du Paléolithique supérieur initial (fig. 3). Enfin, un autre caractère récurrent des ensembles protoaurignaciens concerne la présence d'objets de parure, principalement sous la forme de coquillages perforés (Taborin 1993 ; Bartolomei et al. 1994 ; Kuhn et Stiner 1998 ; Vanhaeren 2002 ; Vanhaeren et d'Errico 2006), trait par ailleurs classique des ensembles culturels du Paléolithique supérieur initial au Proche-Orient (Zilhão 2007). Ici aussi, la remarque faite précédemment à propos de l'implantation géographique de la majeure partie des sites attribués au Protoaurignacien doit être gardée en mémoire.

La recrudescence d'industries à grandes lamelles retouchées occupant une position initiale dans la séquence du Paléolithique supérieur à l'échelle européenne conduit nécessairement à en modifier substantiellement les schémas d'évolution. II semble flagrant que les particularités de tels horizons, connus de longue date, ont peu à peu été gommés pour n'en retenir que les éléments typologiques, tels les lamelles Dufour, supposés les intégrer à la " gran- de famille " aurignacienne. II apparaît pourtant désormais que leur répartition géographique dépasse largement les rivages méditerranéens pour occuper des éco-systèmes diversifiés, rendant compte du succès des idées techniques que ces industries véhiculent. Avant d'essayer d'ordonner et d'interpréter ces éléments, voyons à présent comment l'Aurignacien ancien se définit.

\section{L'Aurignacien ancien et l'exemple du Jura souabe}

L'Aurignacien ancien (Aurignacien I) est, sans conteste, l'épisode le mieux connu puisque c'est à partir de ses outillages lithiques et osseux que l'Aurignacien sensu lato a été individualisé, puis que les phases de son développement ont été articulées (voir p. ex. Breuil 1913 ; SonnevilleBordes 1960). La pointe à base fendue a alors joué un rôle capital puisque partout où elle était présente, de l'Atlantique au Proche-Orient, elle signait l'ancienneté des niveaux en question dans la séquence aurignacienne (Sonneville-Bordes ibid. ; Leroy-Prost 1979 ; Liolios 1999, 2006). C'est précisément à partir des ensembles aurignaciens anciens et la récurrence de leurs caractères (grattoirs carénés, fortes lames à retouche latérale, pointes à base fendue) que l'homogénéité pan-européenne d'un Aurignacien innovant et conquérant a été soutenue.

Plus récemment, des études technologiques réalisées sur les industries lithiques des séquences de référence ont permis de préciser l'unité de l'Aurignacien ancien dans le sud-ouest de la France (voir p.ex. Chiotti 1999 ; Lucas 2000 ; Bon 2002 ; Bordes J.-G. 2006). Ces recherches confiment globalement le découpage typo-chronologique de la séquence aurignacienne et la validité d'un épisode Aurignacien ancien, sans pour autant y surimposer un découpage interne à l'image des anciens faciès Castanet et Ferrassie de l'Aurignacien I (Sonneville-Bordes 1960). Une forte identité technique se dégage des productions lithiques (voir p.ex.Bon 2002 ; Bordes J.-G. 2006), orientées vers la recherche de lames et de lamelles obtenues à partir de chaînes opératoires distinctes. Les lames, volontiers larges et épaisses, sont obtenues par percussion directe tendre à partir de nucléus unipolaires dont la mise en forme demeure en général relativement réduite. Le plus souvent, elles sont destinées à fournir les supports d'un outillage " domestique " (Tartar et al. 2006), fait principalement de grattoirs et de lames retouchées latéralement qui peuvent faire l'objet de plusieurs cycles de ravivage. Les lamelles sont, elles, produites à partir de nucléus spécifiques, en l'occurrence principalement des nucléus carénés à front large. Elles sont plutôt courtes, sub-rectilignes ou courbes et rarement retouchées (Bordes 2006). Lorsque tel est le cas, la retouche semi-abrupte et marg inale est préférentiellement localisée sur le bord droit des supports. Une partie de ces lamelles au moins est dévolue à armer des projectiles (Rigaud 1993 ; Lucas 2000 ; Pelegrin et O'Farrell 2005). A l'Aurignacien ancien, on assiste donc à une individualisation des chaînes opératoires lithiques en fonction des registres d'activité auxquelles elles sont assignées (cf. l' "opposition " domestique vs. chasse). Une même individualisation des sphères fonctionnelles semble également opérante dans le domai- 
ne des matières dures animales, sous l'angle cette fois-ci d'une véritable économie des matières premières (Liolios 1999, 2006 ; Tartar et al. ibid.).

Originellement défini dans le Sud-Ouest français, l'Aurignacien ancien est aussi clairement reconnu dans le Jura souabe, en Allemagne du sud. Dans ce cadre, c'est la grotte de Geissenklösterle qui permet de l'y définir de manière convaincante (voir p.ex. Hahn 1988 ; Conard et Bolus 2003 ; Teyssandier 2003, 2006, 2007 ; Teyssandier et Liolios 2003). Les témoignages les plus anciens y sont documentés dans l'ensemble archéologique III, dont la chronologie a suscité de vives discussions ces demières années (Zilhão et d'Errico 1999, 2003 a et b ; Richter et al. 2000 ; Conard et Bolus 2003 ; Teyssandier 2003, 2006, 2007 ; Teyssandier et al. 2006). La place n'est pas ici de de re-préciser l'interprétation archéo-stratigraphique et chrono-culturelle de cette séquence aurignacienne, déjà présentée plus en détail (Teyssandier ibid. ; Teyssandier et al. ibid.). II convient en revanche d'insister sur la distinction d'au moins deux épisodes principaux d'occupation (de la base vers le sommet, l'ensemble III daté aux alentours de 35 000-32 500 BP et l'ensemble II entre 32500 et 31000 $\mathrm{BP}$ ), attribués à l'Aurignacien ancien tel qu'on le rencontre dans le Sud-Ouest français. Du point de vue de leurs industries lithiques, ils se caractérisent tous deux par une recherche exclusive de lames et de lamelles selon des schémas de production indépendants (fig. 4) : à partir de nucléus unipolaires pour les lames et de nucléus carénés à front large («grattoirs» carénés) pour les lamelles. Cette attribution conjointe des ensembles III et II de Geissenklösterle à l'Aurignacien ancien ne va toutefois pas sans poser de problèmes. En effet, les différences entre ces deux ensembles ne relèvent peut-être pas uniquement de contingences strictement économiques et fonctionnelles. Si l'on peut clairement établir une nette différence dans les modes d'occupation de la grotte entre III, majoritairement un espace de production et II, davantage tourné vers la consommation des produits (Hahn 1988 ; Conard et Bolus 2003 ; Teyssandier 2003 ; Teyssandier et Liolios 2003 ; Teyssandier et al. 2006), d'autres facteurs doivent être pris en considération et, notamment, l'apparition des pointes à base fendue qui s'accompagne d'une gestion différenciée des matières dures animales (Liolios 1999 ; Teyssandier et Liolios 2003) et d'un cortège d'art mobilier en II.

Le fait que ces objets et ces " manières " d'organiser la gestion des productions organiques soient totalement absents des niveaux de base (ensemble III) n'est certainement pas anodin et demande peut-être de repenser quelque peu nos conclusions sur la signification de cette séquence aurignacienne. En effet, dans l'ensemble II de

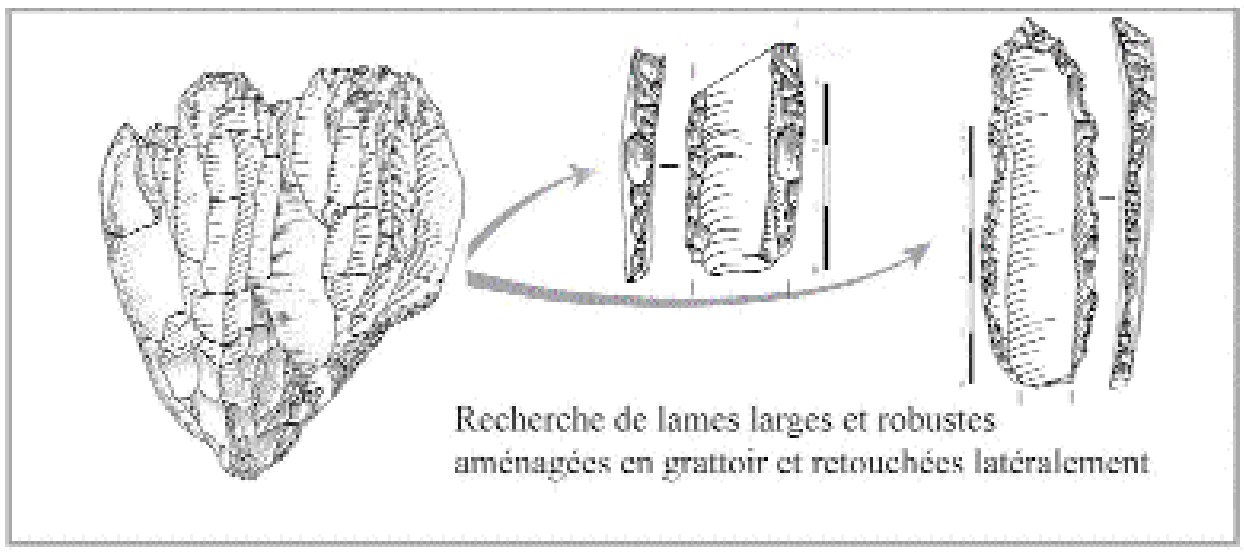

Domination et disjonction des productions laminaires et lamellaires
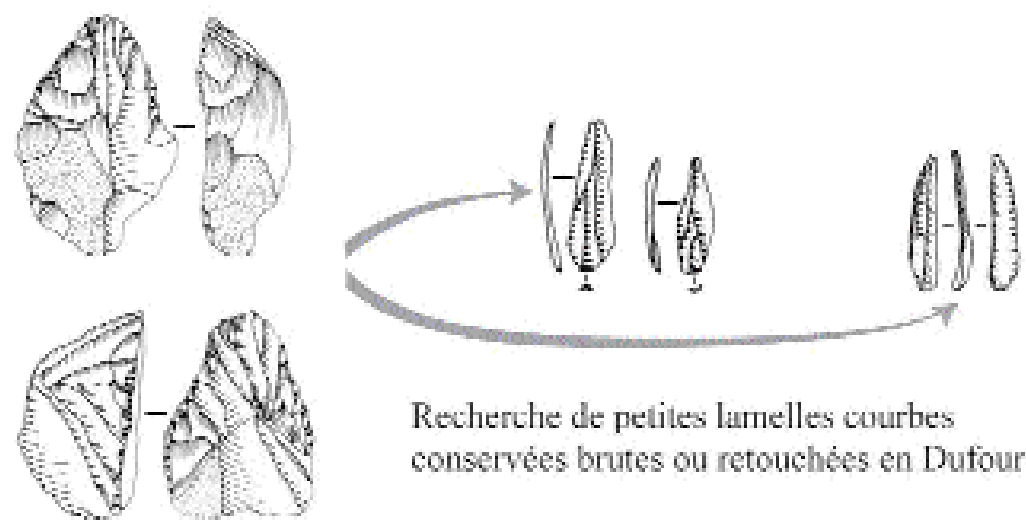

Recherche de petites lamelles courbes conservées brutes ou retouchées en Dufour
Figure 4 - Représentation synthétique de l'organisation des productions lithiques dans l'Aurignacien ancien de Geissenklösterle, Allemagne.

Figure 4 - Synthetic representation of the organization of lithic productions in the Early Aurignacian Geissenklösterle, Germany. 


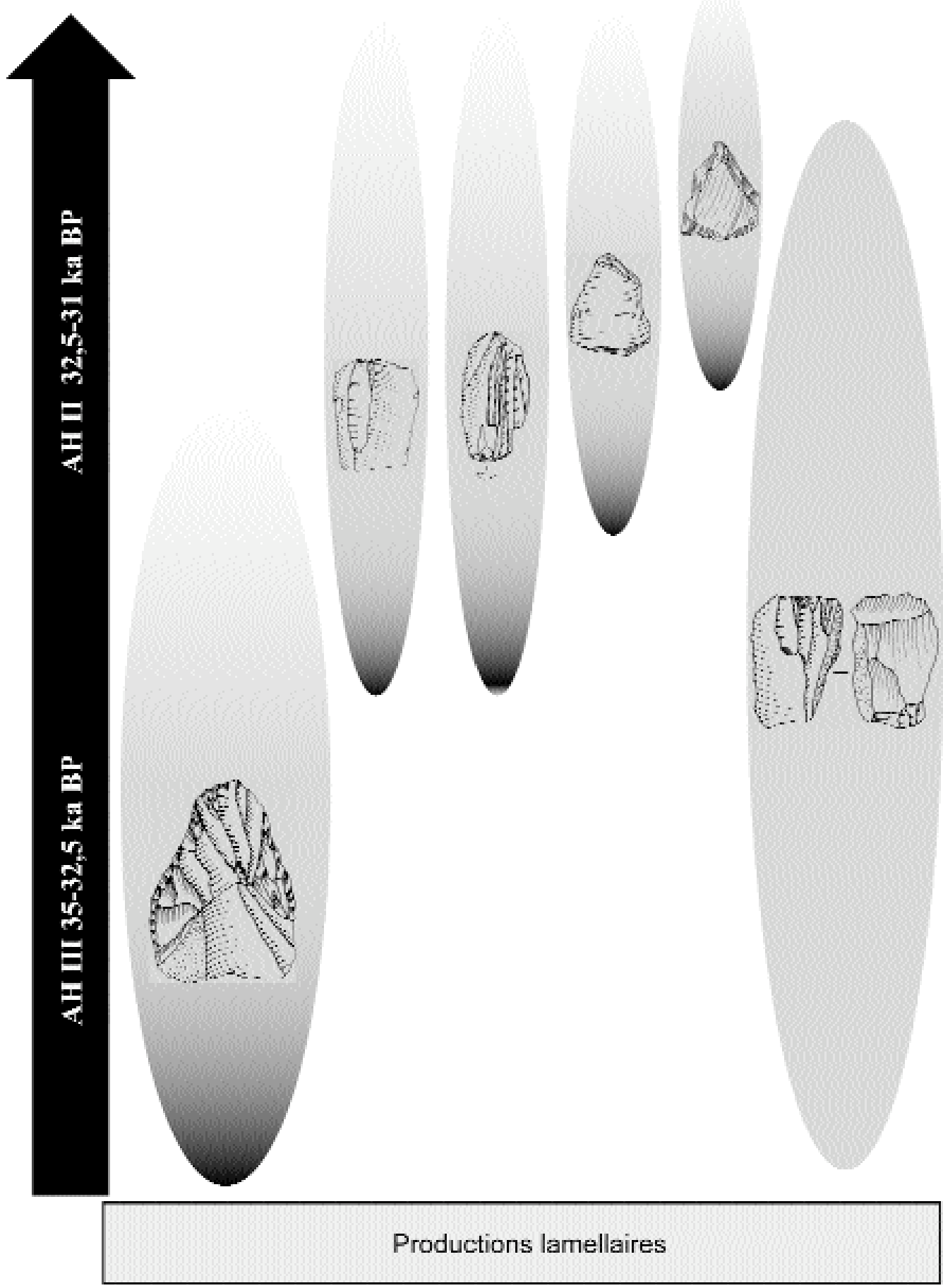

Figure 5 - Représentation synthétique des différents schémas d'exploitation lamellaire et de leur distribution stratigraphique dans la séquence aurignacienne de Geissenklösterle, Allemagne. Cette représentation illustre la plus grande diversité des productions lamellaires et le quasi-abandon des " grattoirs " carénés dans l'ensemble II. Dans ce dernier ensemble, on constate la présence, de gauche à droite, de pièces de Kostienki, de débitages de lamelles intercalées, de burin carénoïde, de grattoir à museau à front étroit et de nucléus à lamelles prismatique. En III, les nucléus ("grattoirs ") carénés sont presque exclusifs.

Figure 5 - Synthetic representation of the bladelet exploitation schemes and their stratigraphic distribution in the Aurignacian sequence of Geissenklösterle, Germany. This representation illustrates the greater diversity of bladelet productions and the near abandon of carinated "scrapers" in AH II. In this latter ensemble, we remark the presence, from left to right, of Kostienki pieces, intercalated bladelet productions, a carinated burin, a nosed scraper with a narrow front and a prismatic bladelet core. In AH III, carinated cores ("scrapers") are nearly exclusive. 
Geissenklösterle, l'apparition des pointes à base fendue coïncide avec la raréfaction des nucléus carénés et une plus grande diversification des schémas de production lamellaire (fig. 5). C'est là une observation quelque peu contradictoire avec les séquences de référence françaises où pointes à base fendue et " grattoirs " carénés à front large sont généralement associés. Une plus grande variabilité pourrait ainsi caractériser l'Aurignacien à pointes à base fendue, idée déjà avancée par $\mathrm{D}$. Liolios pour rendre compte de la diversité dans la conception de ces pointes (Liolios 2006). En outre, l'ensemble III, lui aussi attribué à l'Aurignacien ancien sur la base des caractères de ses productions lithiques, témoigne également d'une certaine originalité vis à vis des séquences ouest-européennes puisque ces mêmes " bases fendues " y sont absentes et que l'on y reconnait la présence de deux pointes en ivoire de section circulaire (fig. $3, n^{\circ}$ 4), qui ne vont pas sans rappeler certains traits de corpus rapportés au Protoaurignacien en France (voir p.ex. la couche VII de la grotte du Renne à Arcy-sur-Cure, fig. $3, \mathrm{n}^{\circ}$ 5 ; Julien et al. 2002, les corpus pyrénéens de Gatzarria et des Abeilles (fig. 3, $n^{\circ} 1-2$; Laplace 1966) ou la couche K du Piage (fig. $3, n^{\circ} 3$; Mons 1981).

L'interprétation de la séquence de Geissenklösterle est rendue délicate par l'absence de points de comparaison fiables et stratifiés en Europe centrale. A ce titre, il faut attendre les publications à venir sur les fouilles en cours du gisement voisin d'Hohle Fels (Conard et Malina 2006), qui semble témoigner d'une succession stratigraphique comparable. Ailleurs en Europe centrale, I'Aurignacien ancien est nettement plus diffus (Teyssandier 2003, 2007). Des ensembles à pointes à base fendue sont c ertes documentés en Slovaquie, Slovénie ou Hongrie, mais ils proviennent le plus souvent de fouilles anciennes et livrent des industries lithiques pauvres et atypiques. Un ré-examen récent des corpus d'Istállóskö (Hongrie) témoigne en outre de mélanges évidents entre des composantes szélétiennes, aurignaciennes et gravetiennes (Teyssandier et al. en préparation). En d'autres termes, rien ne permet d'affirmer que le techno-complexe Aurignacien ancien ait une répartition géographique continue en Europe. Au contraire, il semble bien qu'en dehors de l'Aquitaine, des Pyrénées et du Jura souabe, sa présence soit relativement discrète, tout comme ses caractères intrinsèques sujets à une variabilité inattendue. Des outillages de type Aurignacien ancien et, en particulier, de rares pointes à base fendue découvertes à Kebara et Hayonim, sont certes documentés jusqu'au Proche-Orient (Bar-Yosef 2000) mais le caractère des industries lithiques et l'aspect torse des lamelles de type Dufour évoquent, par comparaison avec les contextes européens, un Aurignacien plus tardif. Cette définition serait en accord avec des datations autour de $32000 \mathrm{BP}$ (Belfer-Cohen et Goring-Morris 2003), plus récentes que celles obtenues pour les contextes Aurignacien ancien à pointes à base fendue en Europe.

Au terme de ce rapide panorama, il apparaît donc un certain décalage entre les données archéologiques présentées et le modèle de référence présidant à l'interprétation de l'Aurignacien.

\section{Le mythe des origines}

L'unité initiale de l'Aurignacien étant désormais très largement discutée, ce sont ses origines qui se complexifient et s'obscurcissent, d'autant plus que, comme le rappellent justement J. Zilhão et F. d'Errico (2003 a), l'imprécision chronologique des dates ${ }^{14} \mathrm{C}$ entre 45000 et $30000 \mathrm{BP}$ rend vaine toute tentative de définir précisément le lieu d'origine et les modalités de la diffusion des principales innovations portées par ces techno-complexes. Mieux vaut donc se prémunir d'une quête effrénée autour de la recherche des origines pour privilégier des recherches inscrites dans le temps long, autour des processus traversant la dite transition du Paléolithique moyen au Paléolithique supérieur.

Dans ce cadre, l'exemple du Bachokirien, longtemps interprété comme un faciès de formation de l'Aurignacien en Europe, s'avère important (voir p. ex. Kozlowski et Otte 2000). Mise en doute dès 1998 (Rigaud 2001), cette interprétation est désormais formellement contredite par les révisions récentes de l'industrie éponyme de la couche 11 de Bacho Kiro (Tsanova et Bordes 2003 ; Teyssandier 2003, 2006, 2007 ; Rigaud et Lucas 2006 ; Tsanova 2006) qui distinguent très clairement cette industrie des normes communes à l'Aurignacien : débitage d'essence Levallois exploitant de larges surfaces de débitage peu convexes par percussion directe dure, recherche de produits convergents laissés bruts ou retouchés en pointes (fig. 6), absence de débitage lamellaire systématisé... La définition technologique du Bachokirien conduit désormais à le comparer à des industries situées à la chamière du Paléolithique moyen au Paléolithique supérieur, qui témoignent d'une certaine leptolithisation des productions lithiques et se cristallisent autour de la recherche de solutions techniques pour produire des supports généralement allongés mais surtout convergents (fig.7), des pointes en l'occurrence (Teyssandier 2003, 2006, 2007 ; Kozlowski 2004 ; Tsanova 2006 ; Zilhão 2006). Tel est le cas en Bulgarie sur le site de Temnata (Drobniewicz et al. 2000 ; Tsanova 2006) mais aussi dans le Bohunicien morave (Skrdla 1996, 2003) et dans de nombreuses séquences du Proche-Orient où ces ensembles sont attribués à un Paléolithique supérieur initial (Marks 1983, 1993 ; Meignen 2007). Il est ainsi envisageable que le Bachokirien s'insère à cette vaste mosaïque qui voit, dès 50 000-45 $000 \mathrm{BP}$, la multiplication de productions laminaires, de conception mixte entre le Levallois et les méthodes annonçant celles du Paléolithique supérieur (Bar-Yosef et Kuhn 1999 ; Skrdla 2003), mais conduites exclusivement par percussion directe dure. Les comparaisons techniques évoquées et la découverte d'ensembles analogues en position géographique intermédiaire entre le Proche-Orient et l'Europe (Üçagizli en Anatolie par exemple, Kuhn et al. 1999, 2001) ont conduit certains auteurs à y voir le résultat de migrations humaines, décalant de fait, dans le temps, la première vague de diffusion des hommes modernes en Europe (Tostevin 2000 ; Kozlowski 2004 ; Bar-Yosef 2006). II nous semble pourtant prématuré de conclure en ce sens, tant cette orientation commune des productions lithiques semble à chaque fois se développer en continuité des substrats locaux pré-existants, comme c'est le cas au Proche-Orient ou dans le 

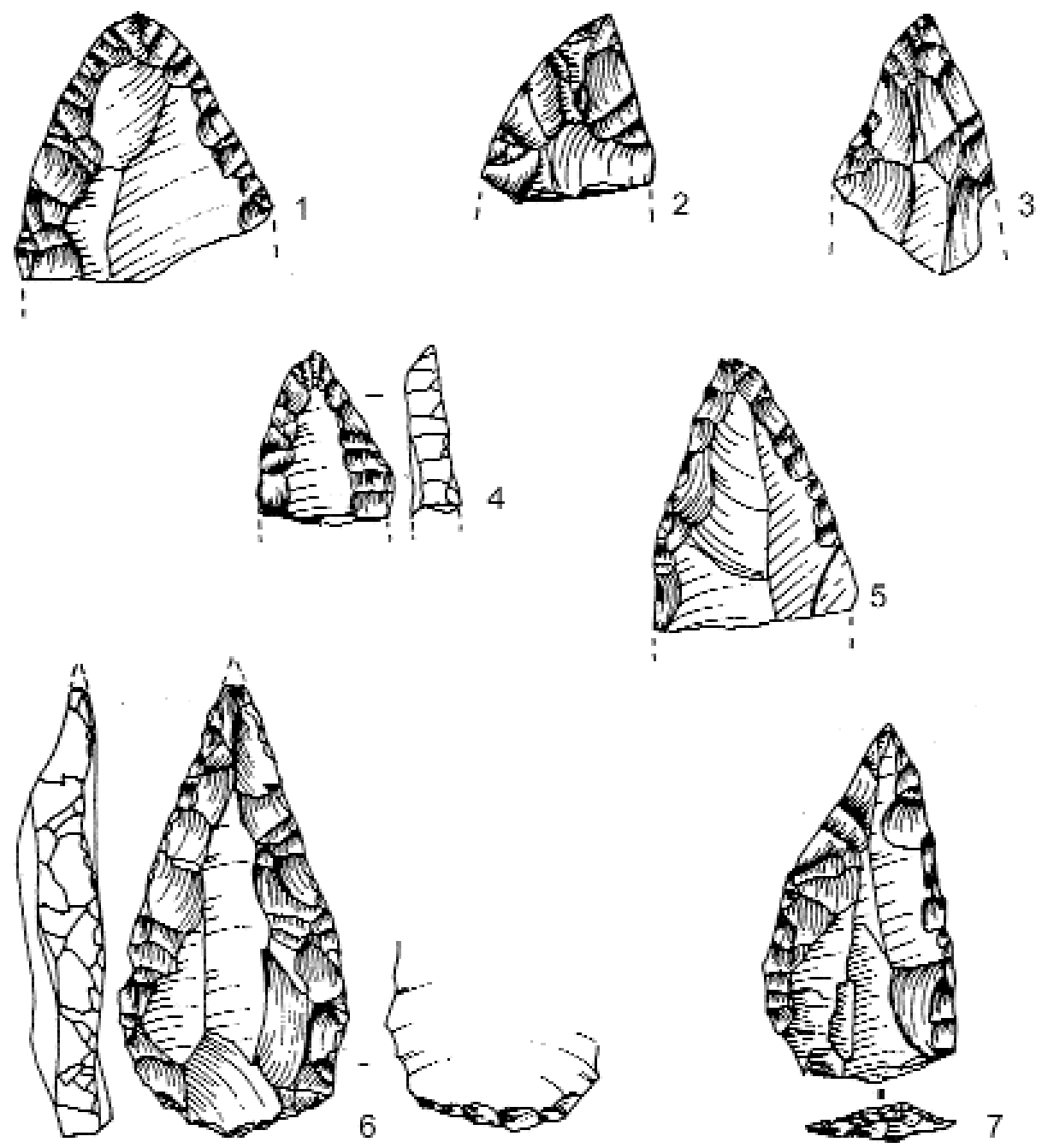

Figure 6 - Pointes retouchées de la couche 11 de Bacho Kiro, Bulgarie (1-7, d'après Kozlowski 1982 ; 8-10, d'après Tsanova 2006 ; 11, d'après Teysandier 2003). Les pièces 1 à 5 et 8 à 11 étaient originellement définies comme des fragments de lames appointées par J.K. Kozlowski ; les nos 6-7 comme des pointes moustériennes jugées intrusives dans l'ensemble de la couche 11. Toutes ces pièces sont désormais interprétées comme des pointes retouchées et noter que les nos 9 et 11 pourraient témoigner de "fractures" complexes. La pièce $n^{\circ}$ 11 présente un enlèvement burinant de $5 \mathrm{~mm}$ de long.

Figure 6 - Retouched points from level 11 of Bacho Kiro, Bulgaria (17, after Kozlowski 1982; 8-10, after Tsanova 2006; 11, after Teysandier 2003).

Pieces 1 to 5 and 8 to 11 were originally defined as fragments of pointed blades by J.K. Kozlowski; 6 and 7 as Mousterian Points judged to be intrusive in the assemblage of level 11. All of these pieces are now interpreted as retouched points and note that nos 9 and 11 could have complex "fractures ". Piece $n^{\circ}$ 11 has a $5 \mathrm{~mm}$ long burin-like removal.
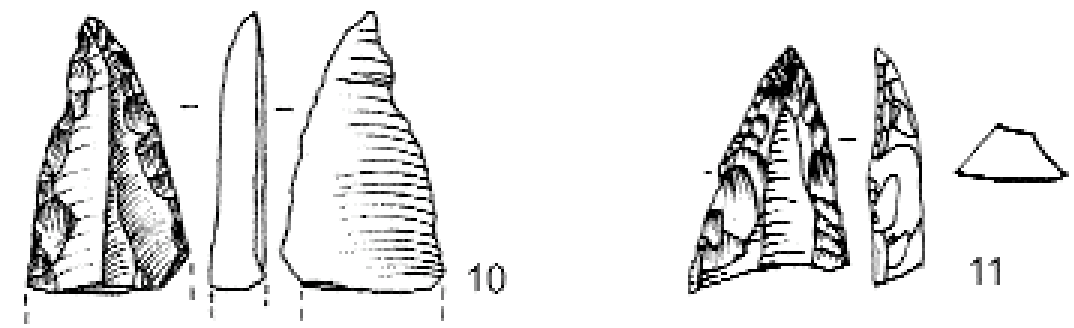

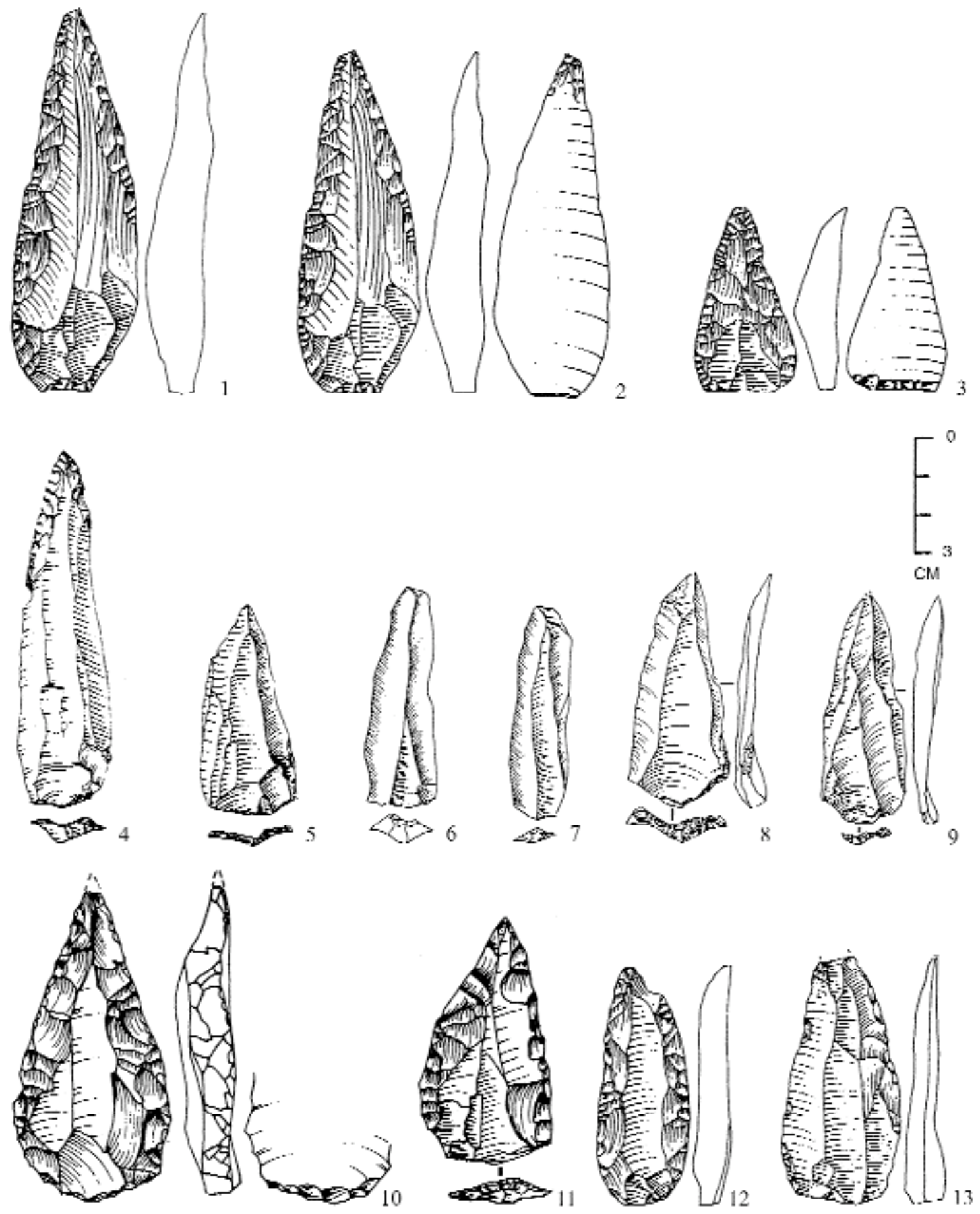

Figure 7 - Comparaison de pointes retouchées et brutes d'industries de transition d'Europe centrale, des Balkans et du Proche-Orient (1 à 9) et du Bachokirien (10 à 13). 1-3: Temnata, TD II, couche VI (d'après Drobniewicz et al. 2000$) ; 4$ et 5 : Bohunicien de Stránská Skála (d'après Svoboda et al. 1996); 6 et 7 : Ksar Aqil, couche XXIV (d'après Meignen 1996); 8 et 9 : Boker Tachtit, couche 2 (d'après Bar-Yosef 2000) ; 10 et 11 : Bacho Kiro, couche 11 (d'après Kozlowski 1982 ) ; 12 et 13 : Temnata, TD I, couche IV (d'après Drobniewicz et al. 2000).

Figure 7 - Comparison of retouched and unretouched points from transitional industries in Central Europe, the Balkans and the Near-East (1 to 9) and the Bachokirian (10 to 13). 1-3: Temnata, TD II, level VI (after Drobniewicz et al. 2000); 4 and 5:

Bohunician from Stránská Skála (after Svoboda et al. 1996); 6 and 7: Ksar Aqil, level XXIV (after Meignen 1996); 8 and 9: Boker Tachtit, level 2 (after Bar-Yosef 2000); 10 and 11: Bacho Kiro, level 11 (after Kozlowski 1982); 12 and 13: Temnata, TD I, level IV (after Drobniewicz et al. 2000). 
Sud-Est français par exemple (Slimak 2004). La rupture que semble opérer ces complexes avec les entités les précédant en Europe centrale ou dans les Balkans pourrait simplement refléter l'important hiatus chronologique les séparant (Zilhão 2006).

\section{Des " armes " comme dénominateur commun ?}

Pour l'heure, force est de constater que parmi la nébuleuse des industries dites de transition se dégage une tendance technique marquée par un relatif allégement des productions et la recherche de supports convergents (fig. 8). Ce " phénomène pointes" semble partout s'ancrer dans des substrats locaux de type Levallois et modifie plus ou moins profondément les systèmes techniques des groupes humains. Mais, dès lors, comment articuler cette mouvance technique avec ce qui lui succédera dans de nombreuses régions : à savoir, la mise en place d'industries (voir p.ex. l'Ahmarien ancien ou le Protoaurignacien) où les productions lamellaires occuperont, pour la premiè re fois, une position structurante des systèmes lithiques.

Dans le cas particulier des productions lithiques, l'un des éléments moteurs des changements qui prennent place à l'aube du Paléolithique supérieur renvoie au rôle dévolu aux éléments de projectile (Bon 2005). Au Protoaurignacien, cette destination fonctionnelle semble être un élément clé de l'orientation des productions autour de l'obtention de lamelles de profil rectiligne dont une part au moins porte des traces caractéristiques d'un usage en projectile (Bon ibid. ; Broglio et al. 2005 ; O'Farrell 2005). Cette nette leptolithisation des industries du Protoaurignacien va s'accentuer à l'Aurignacien ancien et s'accompagner d'une microlithisation des lamelles recherchées qui, désormais, s'individualisent clairement des lames, tant d'un point de vue dimensionnel que par l'indépendance des schémas opératoires présidant à la réalisation de ces deux grandes catégories d'objets. Ainsi, l'une des nouveautés à l'Aurignacien ancien pourrait renvoyer à l'individualisation de deux grands registres d'activités opposant caricaturalement les armes de chasse aux outillages dits domestiques (Tartar et al. 2006). Ce mode d'organisation des productions techniques se vérifierait tant dans le domaine des industries lithiques, à travers l'indépendance des productions de lamelles à partir de " grattoirs " carénés que dans le cadre des productions en matières dures animales, par ailleurs régies selon une stricte économie des matières premières conférant au bois de renne l'exclusivité de la production des éléments d'armement (Liolios 1999).

Ce phénomène de "leptolithisation ", très net dès les débuts de l'Aurignacien, n'est cependant pas totalement nouveau dans la trajectoire évolutive du Paléolithique et il pourrait s'enraciner dans la mosaïque des complexes à pointes le précédant. Ainsi, une préoccupation commune, en l'occurrence la recherche de solutions techniques pour armer des projectiles (Bon 2005) serait un argument pour expliquer l'apparente communauté de ces différents complexes industriels (Emiréen du Proche-Orient, Bachokirien des Balkans, Bohunicien d'Europe centrale, Néronien de la vallée du Rhône...). Cette hypothèse rendrait compte, outre leur orientation technologique commune, de l'importante diversité des " manières de faire " de ces différents complexes, preuve de leur enracinement local dans les industries les précédant. Notons cependant que la destination fonctionnelle de ces pointes n'est pour l'heure pas clairement réglée (Meignen 2007), même si certains auteurs avancent l'idée qu'elles pourraient correspondre à des éléments d'armes d'hast (Shea 2006), comme l'indiqueraient aussi certains stigmates des pointes de la couche 11 de Bacho Kiro (voir fig. 6, $n^{\circ}$ 9, 11 ; Tsanova 2006 ; Teyssandier 2007), qui demanderont des investigations plus poussées.

Ainsi, avant l'éclosion des technologies aurignaciennes, soit globalement entre 45000 et 37000 BP, l'Europe s'apparenterait à une véritable mosaïque de traditions techniques unies dans un même élan, synonyme du bouillonnement d'idées traversant des sociétés humaines généralement présumées néandertalienne par la communauté scientifique. II est en outre intéressant de noter que dans certains de ces complexes (Emiréen ou Bohunicien par exemple), ce changement d'orientation des productions lithiques va conduire certains groupes à repousser les limites intrinsèques du débitage Levallois afin que celui-ci puisse répondre à cet objectif particulier qu'est la production de lames en série (Boëda 2005). Devant cette impossibilité structurelle (Boëda ibid.), les systèmes techniques vont par la suite être conduits à se modifier en profondeur, entraînant l'abandon du concept Levallois et la formalisation de nouveaux modes de taille plus adaptés.

A ce stade, comment ne pas évoquer même brièvement le cas du Châtelperronien, techno-complexe emblématique de ladite transition du Paléolithique moyen au Paléolithique supérieur depuis sa reconnaissance par H. Breuil (1913) comme la première subdivision du Paléolithique supérieur (Aurignacien inférieur) jusqu'à son association à l'Homme de Néandertal (Levêque et Vandermeersch 1980 ; Hublin et al. 1996 ; Bailey et Hublin 2006). Si son aire de répartition géographique se limite à l'Europe occidentale, les orientations techniques qui se dessinent alors vont dans le sens d'une leptolithisation très affirmée puisque les productions lithiques visent très majoritairement l'obtention de lames plutôt courtes et rectilignes principalement dévolues à l'obtention de supports de pointes de Châtelperron (Pelegrin 1995). Des souvenirs moustériens (p.ex. outils sur éclats, racloirs) plus ou moins affirmés selon les séries sont certes documentés mais leur interprétation demeure délicate et leur présence parfois interprétée comme des mélanges avec les industries moustériennes stratigraphiquement sous-jacentes (Rigaud 1996, 2001 ; Bordes 2002). Ainsi, une orientation presque exclusivement tournée vers la production de supports allongés répond à la recherche de produits normés et rectilignes dont une part au moins serait emmanchée pour armer des pointes de sagaies (Pelegrin et Soressi 2007). Là encore, l'idée d'emmancher des traits lithiques modifierait profondément les systèmes techniques à la charnière du Paléolithique moyen et supérieur et ce, indépendamment du développement de l'Aurignacien. Des recherches récentes ont en effet bien montré la stricte antériorité du Châtelperronien sur le premier Aurignacien (d'Errico et al. 1998 ; Rigaud 2001 ; Bordes 2002 ; Pelegrin et Soressi 2007). 

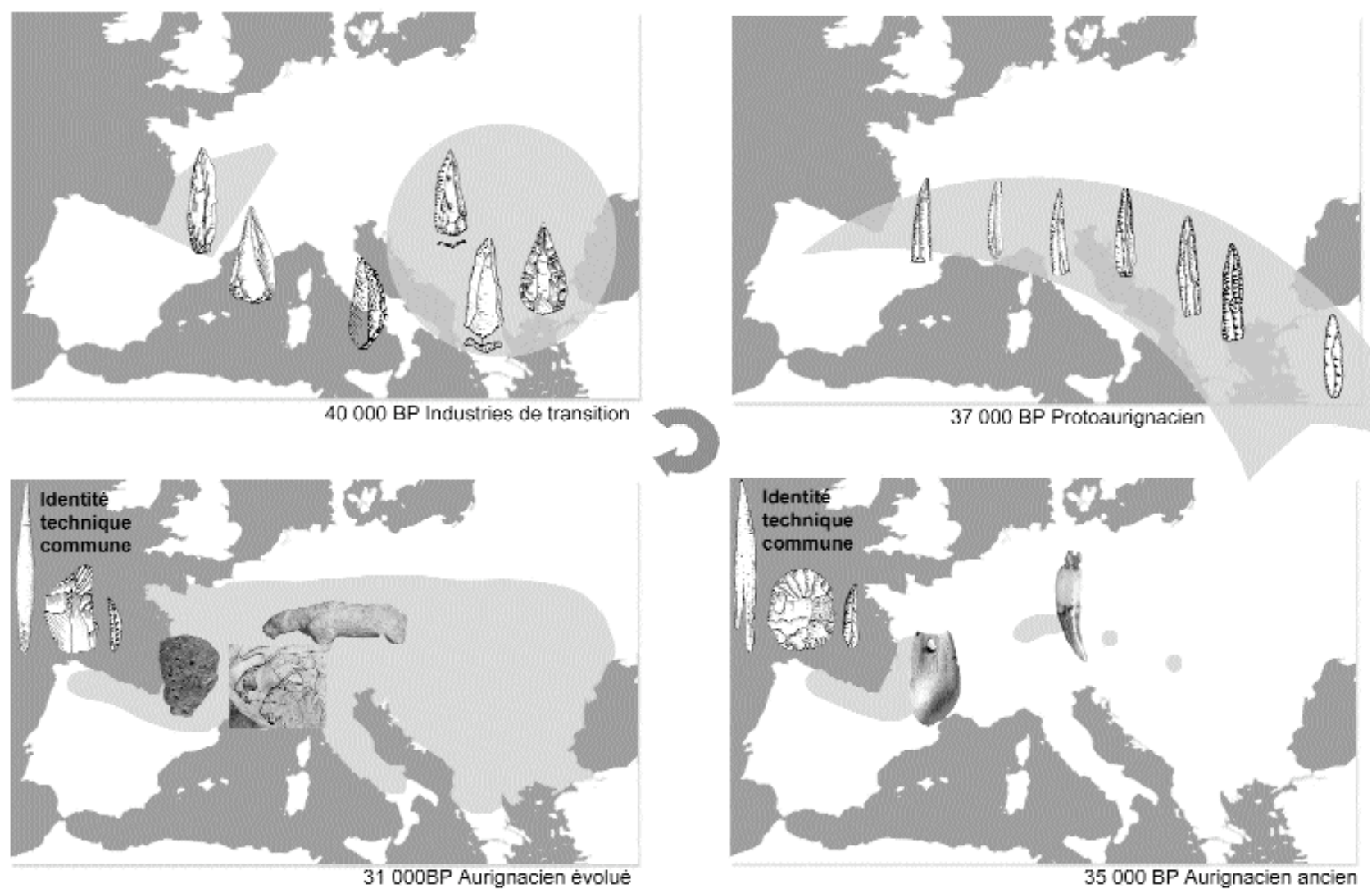

Figure 8 - Représentation schématique de 4 épisodes remarquables s'inscrivant dans les processus conduisant à l'émergence du Paléolithique supérieur. Autour de 40000 BP, une large partie de l'Europe est peuplée par des porteurs de traditions différenciées produisant des pointes (Bachokirien, Bohunicien, Néronien, Châtelperronien...). A partir de $37000 \mathrm{BP}$ environ, apparaît le Protoaurignacien caractérisé par la production de grandes lamelles rectilignes; ses industries présentent des affinités avec l'Ahmarien ancien du Proche-Orient et sont comparables depuis les Balkans jusqu'à la façade atlantique. Ce n'est qu'autour de 35000 BP que, dans certains contextes géographiques, on assiste à l'apparition de l'Aurignacien ancien qui voit des aires régionales se différencier selon le style des parures. Enfin, vers $31000 \mathrm{BP}$, le peuplement aurignacien récent s'étendrait massivement à l'Europe centrale et septentrionale et l'on verrait la naissance d'expressions graphiques mobilières et pariétales.

Figure 8 - Schematic representation of four remarkable episodes in the process leading to the emergence of the Upper Paleolithic. Around 40000 BP, a large part of Europe is occupied by groups with differentiated point producing traditions (Bachokirian, Bohunician, Néronian, Châtelperronian). Starting at around 37000 BP, the Protoaurignacian appears and is characterized by long rectilinear bladelets; these industries present affinities with the Early Ahmarian in the Near East and are comparable from the Balkans to the Atlantic coast. It is not until around $35000 \mathrm{BP}$ that, in certain geographic contexts, the Early Aurignacian appears with regional zones differentiated according to the style of personal ornaments. Finally, at around 31000 $\mathrm{BP}$, the Recent Aurignacian occupation extended massively to central and northern Europe and portable and parietal graphic expressions appear.

Ce n'est que plus tard que la modification des systèmes techniques par une recherche toujours plus soutenue de supports légers, allongés et réguliers se concrétiserait et aboutirait à une relative homogénéisation des traditions techniques à l'échelle de l'Europe et du Proche-Orient. Ce poumait être le cas au Proche-Orient, dans les industries de l'Ahmarien ancien puis, à travers le continent européen, avec les industries du Protoaurignacien. Cela rendrait compte également de la forte communauté technique entre ces deux traditions, désormais soulevée par plusieurs auteurs (Bar-Yosef 2003, 2006 ; Mellars 2006 ; Teyssandier 2006, 2007 ; Tsanova 2006 ; Zilhão 2006) et qu'il s'agira de creuser davantage dans le futur. Cette apparente unification des traditions techniques, transcendant des éco-systèmes variés sur de larges espaces géographiques, pourrait être imputée au vif succès rencontré par les productions lamellaires, sur un plan non seulement techno-fonctionnel pour armer des traits, mais aussi socio-économique, en termes de mobilité pour des groupes de chasseurs-cueilleurs (voir Elston et Kuhn (Eds.) 2002 avec nombreuses références documentées ; Bon 2005). Aux environs de $35000 \mathrm{BP}$, une large part de l'Europe aurait basculé dans le système aurignacien et l'on verrait alors naître, de manière très marquée, des varia- 
tions régionales particulièrement nettes au niveau d'objets ostensibles que sont les parures par exemple (White 1989 ; Vanhaeren 2002 ; Vanhaeren et d'Errico 2006). Ainsi, la longue évolution que représente ce que nous désignons comme "LA " transition pourrait correspondre à une modification des inter-actions sociales entre les groupes humains, débouchant sur la diffusion, à grande échelle, d'idées ou de solutions techniques structurantes. Cette accélération du brassage culturel - et incidemment de celui des gênes - pourrait aller de pair avec un accroissement démographique, souvent avancé dans le cadre du stade isotopique 3 en Europe et concomitant de l'expansion de l'Homme moderne sur ces territoires (voir p.ex. Cachel 1997 ; Shennan 2001 ; Boëda 2005 ; Zilhão 2006). La nécessaire reformulation des rapports sociaux qui s'en suivrait conduirait parallèlement à l'explosion quantitative et à la diversification des productions ostensibles et à l'émergence des procédés graphiques (Zilhão 2007) qui n'allaient, par la suite, cesser de se développer tout au long du Paléolithique supérieur. Ce serait là un demier temps de la " conquête " aurignacienne, comme semblent l'indiquer les dates de Chauvet (Valladas et al. 2005) ou la position stratigraphique et chronologique de l'art mobilier aurignacien en Jura souabe (Verpoorte 2005 ; Teyssandier 2007 ; Zilhão 2007)

\section{Conclusion}

De nombreuses zones d'ombre entourent encore la formulation de ces dernières hypothèses. II convient d'abord de signaler que les acteurs des processus évoqués demeurent encore bien transparents dans la documentation archéologique. Les différentes traditions " à pointes " de la chamière Paléolithique moyen/supérieur (Emiréen, Bachokirien, Bohunicien, Néronien...) n'ont, pour l'heure, pas livré de restes humains, à l'exception d'un fragment de mandibule comprenant une molaire déciduale $(\mathrm{dm} 1)$ découvert à la base de la couche 11 de Bacho Kiro qui ne permet pas de trancher sur le type humain responsable de cette industrie (Churchill et Smith 2000 ; Trinkaus 2005). Habituellement situés dans le groupe des industries dites de transition, ces ensembles sont le plus souvent apparentés aux néandertaliens, suite à la découverte d'une association effective entre le Châtelperronien et ce type humain à Saint-Césaire et Arcy-sur-Cure (Levêque et Vandermeersch 1980 ; Hublin et al. 1996 ; Bailey et Hublin 2006). Rien ne permet pourtant de justifier l'extension de cette association aux industries précédemment évoquées. La situation est tout aussi épineuse pour les débuts de l'Aurignacien. En Europe, les révisions effectuées sur plusieurs spécimens d'hommes anatomiquement modernes présumés contemporains, voire même associés à l'Aurignacien, les ont plus ou moins considéra- blement rajeunis (voir p.ex. Cro-Magnon, Henri-Gambier 2002 ; La Rochette, Orschiedt 2002 ; Hahnöfersand et Kelsterbach, Street et al. 2006 ; Vogelherd, Conard et al. 2004). Les arguments les plus solides dont nous disposons actuellement vont dans le sens d'une association effective de l'Aurignacien récent à Homo sapiens sapiens (voir HenryGambier 2005, et à paraître ; Trinkaus 2005 ;Wild et al. 2005). En revanche, aucune association archéologique indubitable n'existe pour le Protoaurignacien ${ }^{2}$ et la situation n'est pas beaucoup plus favorable en ce qui concerne l'Aurignacien ancien où seuls des corpus dentaires ne faisant pas consensus quant à leur attribution sont connus (voir p.ex. HenryGambier et al. 2004 ; Bailey et Hublin 2005). En définitive, ce ne sont que quelques données encore bien parcellaires qui soutiennent l'idée d'une association entre l'Aurignacien au sens large du terme et l'Homme moderne. Cependant, et même si cette parenté devait être confirmée par des recherches à venir, cela ne renforcerait pas nécessairement la validité de la seule théorie du remplacement et de son corrélat culturel percevant une rupture radicale entre les derniers temps du Paléolithique moyen et les premières expressions du Paléolithique supérieur. Nos propositions mettent davantage l'accent sur le caractère progressif et graduel de la mise en place des traits constitutifs du Paléolithique supérieur.

De ce point de vue, plusieurs consensus restent à établir :

- le premier renvoie à la position d'antériorité des industries à grandes lamelles retouchées (Protoaurignacien, Ahmarien ancien) vis-à-vis de celles à " grattoirs " carénés et pointes à base fendue (Aurignacien ancien). Cela s'obsene d'abord au travers de plusieurs cas de successions stratigraphiques en Europe occidentale : Le Piage (Bordes J.-G. 2002, 2003), Isturitz (Normand 2006), Gatzarria, (Laplace 1966), Les Abeilles (Laplace 1966 ; Laplace et al. 2006 ; Eizenberg 2006) et Labeko Koba (Arrizabalaga 2006). Les séquences proche-orientales et les dates anciennes qui y ont été obtenues pour l'Ahmarien ancien (Goring-Moms et Belfer-Cohen (Eds.) 2003), ainsi que les mesures ${ }^{14} \mathrm{C}$ récemment publiées pour la couche VII de Kozamika en Bulgarie (Guadelli et al. 2005 ; Tsanova 2006) comoborent ces observations ;

- le second tient à l'emprise géographique du phénomène " grandes lamelles retouchées", désormais connu, outre sa zone de reconnaissance initiale, en Aquitaine, en Bourgogne mais aussi en Autriche et en Roumanie et ce, jusque dans les Balkans. D'une tradition restreinte aux régions ouest-méditerranéennes, nous l'envisageons dés o mais comme une entité pan-européenne, d'autant plus que des comparaisons poussent chaque jour à

(2) Plusieurs auteurs (Mellars 2006 ; Zilhão 2006, 2007) ont récemment proposé que les industries à grandes lamelles retouchées du Paléolithique supérieur initial (Ahmarien ancien, Protoaurignacien) soient le produit de l'Homme modeme. Ceci repose sur des faisceaux d'arguments constitués notamment par la découverte de restes humains indubitablement modemes, datés aux alentours de 35000 BP à Oase (Roumanie), sans aucun contexte culturel mais non loin du site de Tincova dont l'outillage lithique permet des comparaisons avec le Protoaurignacien (Zilhão 2006 ; Teyssandier 2007). De même, une association Ahmarien ancien/Homme moderne est de longue date évoquée à partir des restes d'un individu juvénile (Egbert) découvert dans le niveau 17 de Ksar Aqil au Liban (Ewing 1947 ; Bergman 2003 ; Trinkaus 2005). Ce squelette a hélas disparu, ôtant tout espoir d'essayer de le dater directement par AMS. 
accroître l'ampleur du phénomène, nous conduisant jusqu'au Proche-Orient voire même en Iran (voir p.e. le cas du Baradostien, Otte et Kozlowski 2004 ; Jaubert et al. 2005). Ici, il importe de constater que la pleine expression de l'Aurignacien ancien à pointes à base fendue qui, il y a peu, consacrait l'aspect conquérant de cette avancée de l'Homme moderne, se trouve restreinte à une portion " occidentale " de l'Europe (Aquitaine, Pyrénées, Jura souabe). En ce sens, et compte-tenu de sa postériorité vis-à-vis du Protoaurignacien, l'Aurignacien ancien semble bien représenter un phénomène d'essence européenne (Teyssandier 2006, 2007), né des basculements opérés précédemment par les porteurs d'autres traditions techniques ;

- enfin, le troisième renvoie à la manière dont nous pouvons essayer de structurer l'évolution interne de l'Aurignacien. La perception d'innovations soudaines et brutales est désormais discutée au profit d'une apparition plus graduelle des éléments constitutifs de ce Paléolithique supérieur émergeant. Ce pourrait être vrai pour les productions lithiques (voir p.ex. l'individualisation progressive des productions laminaires et lamellaires), mais aussi pour l'exploitation technique et symbolique des matières dures animales ou les manifestations graphiques qui n'apparaîtraient durablement qu'aux alentours de 32 000-31 000 BP (Valladas et al. 2005 ; Zilhão 2007). In fine, une certaine arythmie présiderait à la mise en place des caractères constitutifs du Paléolithique supérieur (Teyssandier 2007).

Notre propos, le lecteur l'aura compris, ne veut pas ici atténuer l'ampleur des transformations qui prennent place avec le Paléolithique supérieur. Celles-ci sont évidentes et touchent à tous les domaines de la vie de ces sociétés humaines. Nous avons en revanche souhaité mettre l'accent sur l'aspect plus graduel et progressif de la mise en place des traits définissant en général le Paléolithique supérieur. Ceci impose désormais de re-discuter des scénarios classiques de son apparition.

II nous faut aussi penser l'hétérogénéité potentielle de l'histoire des sociétés humaines du Paléolithique supérieur. L'Aurignacien ne doit plus être considéré comme une étape obligatoire sur les chemins de l'évolution du Paléolithique européen, donnée appuyée par les recherches conduites récemment au sein d'espaces où, auparavant, nos données étaient bien indigentes (voir p.ex. Brantingham et al. (éds.) 2004 ; Bar-Yosef et al. 2006 ; Flas 2006). Parmi d'autres exemples possibles, citons l'absence des phases anciennes de l'Aurignacien et le développement, entre 38000 et $35000 \mathrm{BP}$, des traditions de type Lincombien-Ranisien-Jerzmanowicien héritées du Paléolithique moyen récent dans les plaines septentrionales de l'Europe (Flas 2006), qui n'empêcheront en rien ces régions de connaître par la suite le développement d'un Paléolithique supérieur "véritable ". C'est dont à un renouvellement des scénarios et à un "retour" au terrain que doivent maintenant se consacrer les travaux sur cette période phare de l'évolution des sociétés humaines.

\section{Remerciements}

François Bon a contribué à faire avancer ma réflexion sur les thèmes abordés dans cet article. Je le remercie ainsi que J.-G. Bordes et J. Zilhão pour les discussions menées ensemble. Je remercie aussi sincèrement J.-Ph. Rigaud pour sa relecture critique et ses commentaires. De nombreux collègues et amis m'ont apporté un soutien moral important durant la rédaction de cet article. II serait trop long de tous les remercier ici mais qu'ils sachent que je n'oublie pas leur témoignage. Enfin, une pensée émue en mémoire de Chantal Fortin dont la gentillesse et la générosité nous manqueront lors de nos passages au Musée National de Préhistoire des Eyzies-de-Tayac.

\section{BIBLIOGRAPHIE}

ARRIZABALAGA A. 2006 - La industria lítica del yacimiento del Paleolítico de Labeko Koba (País Vasco). In : F. Bon, J.M. Maillo Fernandez et D. Ortega i Cobos (Eds.), Autour des concepts de Protoaurignacien, d'Aurignacien archaïque, initial et ancien. Unité et variabilité des comportements techniques des premiers groupes d'hommes modernes dans le Sud de la France et le Nord de I'Espagne. Madrid, UNED, p. 117-134, ( Serie I, Prehistoria y Arqueologia, 15).

BAILEY S.E., HUBLIN J.-J. 2005 - Who made the early Aurignacian? A reconsideration of the Brassempouy dental remains. Bulletins et Mémoires de la Société d'Anthropologie de Paris, 17, p. 115-121.

BAILEY S.E., HUBLIN J.-J. 2006 - Dental remains from the Grotte du Renne at Arcy-sur-Cure (Yonne). Journal of Human Evolution, 50, p. 485-508.

BAR-YOSEF O. 2000 - The Middle and Early Upper Paleolithic in Southwest Asia and neighboring regions. In : O. Bar-Yosef et D. Pilbeam (Eds.), The geography of Neandertals and Modern Humans in Europe and the greater Mediterranean. Cambridge, Harvard University, p. 107-156.

BAR-YOSEF O. 2003 - Away from home : prehistoric colonizations, exchanges and diffusions in the Mediterranean basin. In : B. Vandermeersch (Ed.), Echanges et diffusion dans le préhistoire méditerranéenne. Paris, CTHS, p. 71-81.

BAR-YOSEF O. 2006 - Le cadre archéologique de la révolution du Paléolithique supérieur. Diogène, 214, p. 3-23.

BAR-YOSEF O., KUHN S.L. 1999 - The big deal about blades : laminar technologies and human evolution. American Anthropologist, 101, p. 332-338.

BAR-YOSEF O., BELFER-COHEN A., ADLER D. 2006 The implications of the Middle-Upper Paleolithic chronological boundary to eurasian prehistory Anthropologie, XLIV, 1, p. $49-60$. 
BARTOLOMEI G., BROGLIO A., CASSOLI P.F., CASTELLETTI L., CATTANI L., CREMASCHI M., GIACOBINI G., MALERBA G., MASPERO A., PERESANI M., SARTORELLI A., TAGLIACOZZO A. 1994 - La grotte de Fumane. Un site aurignacien au pied des Alpes. Preistoria Alpina, 28, 1, p. 131-179.

BAZILE F. 1974 - Nouvelles données sur le Paléolithique supérieur ancien en Languedoc oriental. In : Congrès Préhistorique de France. Paris, Société Préhistorique de France, p. 24-28.

BAZILE F. 2006 - Le premier Aurignacien en France méditerranéenne. Un bilan. In : F. Bon, J.M. Maillo Fernandez et D. Ortega i Cobos (Eds.), Autour des concepts de Protoaurignacien, d'Aurignacien archaïque, initial et ancien. Unité et variabilité des comportements techniques des premiers groupes d'hommes modernes dans le Sud de la France et le Nord de l'Espagne. Madrid, UNED, p. 215-236, ( Serie I, Prehistoria y Arqueologia, 15).

BELFER-COHEN A., GORING-MORRIS N. 2003 - Current issues in Levantine Upper Palaeolithic research. In : More than Meets the Eye : Studies on Upper Paleolithic diversity in the Near East. Oxford, Oxbow Books, p. 1-12.

BERGMAN C.A. 2003 - Twisted debitage and the Levantine Aurignacian problem. In : N. Gorring-Morris et A. Belfer-Cohen (Eds.), More than meets the eye. Oxford, Oxbow Books, p. 185-195.

BOËDA E. 2005 - Paleo-technologie ou anthropologie des techniques ? Arob@se, 1, p. 46-64.

BON F. 2002 - L'Aurignacien entre mer et océan. Réflexion sur l'unité des phases anciennes de l'Aurignacien dans le sud de la France. Paris, Société Préhistorique Française, (Mémoire, 29).

BON F. 2005 - Little big tool. Enquête autour du succès de la lamelle. In : F. Le Brun-Ricalens (Ed.), Productions lamellaires attribuées à l'Aurignacien, chaînes opératoires et perspectives technoculturelles. Luxembourg, MNHA, p. 479-484, ( ArchéoLogiques, 1).

BORDES F. 1963 - À propos de la théorie de M. Laplace sur le synthétotype aurignaco-gravettien. Quelques questions préalables. L'Anthropologie, 67, 3-4, p. 347-360.

BORDES J.-G. 2002 - Les interstratifications Châtelperronien/Aurignacien du Roc-de-Combe et $d u$ Piage (Lot, France). Analyse taphonomique des industries lithiques ; implications archéologiques. Thèse de doctorat, Université Bordeaux 1.

BORDES J.-G. 2003 - Lithic taphonomy of the Châtelperronian/Aurignacian interstratifications in Roc de Combe and Le Piage (Lot, France). In : J. Zilhão et F. d'Errico (Eds.), The chronology of the Aurignacian and of the transitional technocomplexes. Dating, stratigraphies, cultural implications. Lisbonne, Instituto Português de Arqueologia, p. 223-244, (Trabalhos de Arqueologia, 33).
BORDES J.-G. 2006 - News from the West: a reevaluation of the classical Aurignacian sequence of the Perigord. In : O. Bar-Yosef et J. Zilhão (Eds.), Towards a definition of the Aurignacian. Lisbonne, Instituto Português de Arqueologia, p. 147-171, (Trabalhos de Arqueologia, 45).

BRANTINGHAM P.J., KUHN S.L., KERRY K.W. (Eds.) 2004 The Early Upper Paleolithic beyond Westem Europe. Berkeley, University of California Press.

BREUIL H. 1913 - Les subdivisions du Paléolithique supérieur et leur signification. Congrès International d'Anthropologie et d'Archéologie Préhistorique. Genève, 1, p. 165-223.

BROGLIO A., BERTOLA S., DE STEFANI M., MARINI D., LEMORINI C., ROSSETTI P. 2005 - La production lamellaire et les armatures lamellaires de l'Aurignacien ancien de la grotte de Fumane (Monts Lessini, Vénétie). In : F. Le Brun-Ricalens (Ed.), Productions lamellaires attribuées à l'Aurignacien, chaînes opératoires et perspectives technoculturelles. Luxembourg, MNHA, p. 415-436 (ArchéoLogiques, 1).

CABRERA VALDÈS V., MAILLO J.M., LLORET M., BERNALDO DE QUIROS F. 2001 - La transition vers le Paléolithique supérieur dans la grotte du Castillo (Cantabrie, Espagne) : la couche 18. L'Anthropologie, 105, p. 505-532.

CACHEL S. 1997 - Dietary shift and the European Upper Paleolithic transition. Current Anthropology, 38, p. 579-603.

CHIOTTI L. 1999 - Les industries lithiques des niveaux aurignaciens de l'abri Pataud, Les Eyzies-de-Tayac (Dordogne) : étude technologique et typologique. Thèse de doctorat, Muséum d'Histoire Naturelle, Institut de Paléontologie Humaine.

CHURCHILL S.E., SMITH F.H. 2000 - Makers of the early Aurignacian of Europe. Yearbook of Physical Anthropology, 43, p. 61-115.

CLARK G.A., LINDLY J.M. 1990 - The case for continuity : obsenations on the biocultural transition in Europe and Westem Asia. In : P. Mellars (Ed.), The emergence of Modem Humans. An archaeological perspective. Edinburgh, Edinburgh University Press, p. 626-676.

CONARD N.J., BOLUS M. 2003 - Radiocarbon dating the appearance of modern humans and timing of cultural innovations in Europe : new results and new challenges. Joumal of Human Evolution, 44, p. 331-371.

CONARD N.J., MALINA M. 2006 - Neue Ergebnisse zum Mittelpaläolithikum, zum Aurignacien und zu den letzen Neandertalemam Hohle Fels bei Schelklingen, Alb Donau Kreis. Archäologische Ausgrabungen in BadenWürttemberg, 2005, p. 17-20.

CONARD N.J., GROOTES P.M., SMITH F.H. 2004 Unexpectedly recent dates for human remains from Vogelherd. Nature, 430, p. 198-201. 
DAVIES W. 2001 - A very model of a Modern Human industry : new perspectives on the origins and spread of the Aurignacian in Europe. Proceedings of the Prehistoric Society, 67, p. 195-217.

DEMARS P.-Y., HUBLIN J.-J. 1989 - La transition néandertaliens/Hommes de type moderne en Europe occidentale : aspects paléontologiques et culturels. In : B. Vandemeersch (Ed.), L'Homme de Neandertal. L'extinction. Liège, ERAUL 34, p. 23-37.

DEMARS P.-Y., LAURENT P. 1989 - Types d'outils lithiques du Paléolithique supérieur en Europe. Paris, Éditions du CNRS (Cahiers du Quatemaire, 14).

D'ERRICO F., ZILHÃO J., JULIEN M., BAFFIER D., PELEGRIN J. 1998 - Neanderthal acculturation in Westem Europe ? A critical review of the evidence and its interpretation. Current Anthropology, 39, p. S1-S44.

DJINDJIAN F. 1993 - Les origines du peuplement aurignacien en Europe. In : L. Bánesz et J.K. Kozlowski (Eds.), Aurignacien en Europe et au Proche-Orient. Bratislava, Institut Archéologique de l'Académie Slovaque des Sciences, p. 136-154.

DJINDJIAN F. 2006 - Cinquante années de recherche sur les débuts de l'Aurignacien en Europe occidentale. In : F. Bon, J.M. Maillo Fernandez et D. Ortega i Cobos (Eds.), Autour des concepts de Protoaurignacien, d'Aurignacien archaiqque, initial et ancien. Unité et variabilité des comportements techniques des premiers groupes d'hommes modernes dans le Sud de la France et le Nord de l'Espagne. Madrid, UNED, p. 17-38, ( Serie I, Prehistoria y Arqueologia, 15).

DROBNIEWICZ B., GINTER B., KAZIOR B., KOZLOWSKI J.K. 2000 - "Transitional" industry from layer VI, trench TD-II. In : B. Ginter, J.K. Kozlowski, J.-L. Guadelli et H. Laville (Eds.), Temnata cave, excavations in Karlukovo karst area, Bulgaria. Cracovie, Jagellonian University, p. 243-316.

EIZENBERG L. 2006 - Le rôle de l'Aurignacien archaïque dans les phases initiales du Paléolithique supérieur: apports des industries lithiques de la grotte des Abeilles (Haute-Garonne). Mémoire de Master 2, Université Toulouse Le Mirail.

ELSTON R.G., KUHN S.L. (Eds.) 2002 - Thinking small : global perspectives on micolithization. Arlington, American Anthropological Association, (Archeological Papers of the American Anthropological Association, 12).

EWING J.F. 1947 - Preliminarynote on the excavations at the Paleolithic site of Ksar Aqil, Republic of Lebanon. Antiquity, 21, p. 186-196.

FLAS D. 2006 - La transition du Paléolithique moyen au Paléolithique supérieur dans la plaine septentrionale de l'Europe. Les problématiques du Lincombien-RanisienJerzmanowicien. Thèse de doctorat, Université de Liège.
GORING-MORRIS N., BELFER-COHEN A. (Eds) 2003 More than Meets the Eye : Studies on Upper Paleolithic diversity in the Near East. Oxford, Oxbow Books.

GUADELLI J.-L., SIRAKOV N., IVANOVA S., SIRAKOVA S., ANASTASSOVA E., COURTAUD P., DIMITROVA I., DJABARSKA N., FERNANDEZ P., FERRIER C., FONTUGNE M., GAMBIER D., GUADELLI A., IORDANOVA D., IORDANOVA N., KOVATCHEVA M., KRUMOV I., LEBLANC J.-CL., MALLYE J.-B., MARINSKA M., MITEVA V., POPOV V., SPASSOV R., TANEVA S., TISNERATLABORDE N., TSANOVA T. 2005 - Une séquence du Paléolithique inférieur au Paléolithique récent dans les Balkans : la grotte Kozarnika à Orechets (Nord-Ouest de la Bulgarie). In : N. Molines, M.-H. Moncel et J.-L. Monnier (Eds.), Les premiers peuplements en Europe. Données récentes sur les modalités de peuplement et sur le cadre chronostratigraphique, géologique et paléogéographique des industries du Paléolithique ancien et moyen en Europe. Oxford, John and Erica Hedges Ltd., p. 87-103 (BAR Intemational Series, 1364).

HAHN J. 1977 - Aurignacien, das ältere Jungpaläolithikum in Mittel- und Osteuropa. Köln/Wien, Böhlau-Verlag.

HAHN J. 1988 - Die Geißenklösterle-Höhle im Achtal bei Blaubeuren I. Fundhorizontbildung und Besiedlung im Mittelpaläolithikum und im Aurignacien. Stuttgart, Konrad Theiss Verlag.

HENRY-GAMBIER D. 2002 - Les fossiles de Cro-Magnon (Les Eyzies-de-Tayac, Dordogne) : nouvelles données sur leur position chronologique et leur attribution culturelle. Paléo, 14, p. 201-204.

HENRY-GAMBIER D. 2005 - Les populations européennes du Paléolithique supérieur. In : O. Dutour, J.-J. Hublin et B. Vandermeersch (Eds.), Origine et évolution des populations humaines. Paris, CTHS, p. 151-176.

HENRY-GAMBIER D. à paraître - Le peuplement Aurignacien de l'Europe. In : H. Floss et $\mathrm{N}$. Rouquerol (Eds.), Les chemins de l'art aurignacien.

HENRY-GAMBIER D., MAUREILLE B., WHITE R. 2004 Vestiges humains des niveaux de l'Aurignacien ancien du site de Brassempouy (Landes). Bulletins et Mémoires de la Société d'Anthropologie de Paris, 16, p. 49-87.

HUBLIN J.-J., SPOOR F., BRAUN M., ZONNEVELD F., CONDEMI S. 1996 - A late Neanderthal associated with Upper Paleolithic artefacts. Nature, 381, p. 224-226.

JAUBERT J., BIGLARI F., BORDES J.-G., BRUXELLES L., MOURRE V., SHIDRANG S., NADERI R., ALIPOUR S. 2005 - New researches on Paleolithic of Iran : preliminary report of 2004 Iranian-French joint mission. Archaeological Reports, 4, ICHTO, Téhéran, p. 18-26.

JULIEN M., BAFFIER D., LIOLIOS D. 2002 - L'outillage en matières dures animales. In : B. Schmider (Ed.), 
L'Aurignacien de la grotte du Renne. Les fouilles d'André Leroi-Gourhan à Arcy-sur-Cure (Yonne). Paris, CNRS Éditions, p. 217-250, (34 ${ }^{\mathrm{e}}$ supplément à Gallia Préhistoire).

KOZLOWSKI J.K. 1993 - L'Aurignacien en Europe et au Proche-Orient. In : L. Bánesz et J.K. Kozlowski (Eds.), Aurignacien en Europe et au Proche-Orient. Bratislava, Institut Archéologique de l'Académie Slovaque des Sciences, p. 283-291.

KOZLOWSKI J.K. 2004 - Early Upper Paleolithic Levalloisderived industries in the Balkans and in the Middle Danube basin. Anthropologie, XLII/3, p. 263-280.

KOZLOWSKI J.K., OTTE M. 2000 - The formation of the Aurignacian in Europe. Joumal of Anthropological Research, 56, p. 513-534.

KUHN S.L., STINER M.C. 1998 - The earliest Aurignacian of Riparo Mochi (Liguria, Italy). Current Anthropology, 39, 3, p. 175-189.

KUHN S.L., STINER M.C., GÜLEC E. 1999 - Initial Upper Paleolithic in South-Central Turkey and ist regional context. Antiquity, 73, p. 505-517.

KUHN S.L., STINER M.C., REESE D.S. GÜLEC E. 2001 O maments of the earliest Upper Paleolithic : new insights from the Levant. Proceedings of the National Academy of Sciences USA, 98, p. 7641-7646.

LAPLACE G. 1966 - Recherches sur l'origine et l'évolution des complexes leptolithiques. Paris, Éditions de Boccard, (Mélanges d'Archéologie et d'Histoire, École Française de Rome, $4^{\mathrm{e}}$. supplément).

LAPLACE G., BARANDIARAN I., SAENZ DE BURUAGA A., ALTUNA J. 2006 - Les Aurignaciens pyrénéens des Abeilles et méditerranéen de Régismont-le-Haut. Analyses typologique et paléontologique. Les Eyzies-de-Tayac, SAMRA.

LEROY-PROST C. 1979 - L'industrie osseuse aurignacienne. Essai régional de classification : Poitou, Charentes, Périgord. Gallia Préhistoire, 22, 1, 205-370.

LÉVÊQUE F., VANDERMEERSCH B. 1980 - Découverte de restes humains dans un niveau castelperronien à SaintCésaire (Charente-Maritime). Comptes Rendus de l'Académie des Sciences de Paris, 291, p. 187-189.

LIOLIOS D. 1999 - Variabilité et caractéristiques du travail des matières osseuses au début de l'Aurignacien : approche technologique et économique. Thèse de doctorat, Université Paris X-Nanterre.

LIOLIOS D. 2006 - Reflections on the role of bone tools in the definition of the Early Aurignacian. In : O. Bar-Osef et J. Zilhão (éds.), Towards a definition of the Aurignacian. Lisbonne : Instituto Poruguese de Arqueologia : 37-51, (Trabalhos de Arqueologia, 45).
LUCAS G. 2000 - Les industries lithiques du Flageolet I (Dordogne) : approche économique, technologique et fonctionnelle. Thèse de doctorat, université Bordeaux I.

MAÍLLO FERNÁNDEZ J.M. 2005 - La production lamellaire de l'Aurignacien de la grotte Morin (Cantabrie, Espagne). In : F. Le Brun-Ricalens (Ed.), Productions lamellaires attribuées à l'Aurignacien, chaînes opératoires et perspectives technoculturelles. Luxembourg, MNHA, p. 339-358 ( ArchéoLogiques, 1)

MARKS A.E. (Ed.) 1983 - Prehistory and palaeoenvironments in the Central Negev, Israel. Dallas, Southern Methodist University Press.

MARKS A.E. 1993 - The Early Upper Paleolithic : the view from the Levant. In : H. Knecht, A. Pike-Tay et R. White (Eds.), Before Lascaux : the complex record of the Early Upper Paleolithic. Boca Raton, CRC Press, p. 5-22.

MAROTO J., SOLER N., FULLOLA J.M. 1996 - Cultural changes between Middle and Upper Palaeolithic in Catalonia. In : E. Carbonell et M. Vaquero (Eds.), The last Neandertals, the first Anatomically Modern Humans : a tale about the human diversity. Cultural change and human evolution : the crisis at $40 \mathrm{ka}$ BP. Tarragona, Universitat Rovira i Virgili, p. 219-250.

MEIGNEN L. 2007 - Néandertaliens et Hommes modemes au Proche-Orient : connaissances techniques, stratégies de subsistance et mobilité. In : B. Vandemeersch et B. Maureille (Eds.), Les Néandertaliens, biologie et cultures. Paris, CTHS, p. 231-261, (Documents Préhistoriques $n^{\circ} 23$ ).

MELLARS P. 1989 - Major issues in the emergence of modem humans. Current Anthropology, 30, p. 349-385.

MELLARS P. 2004 - Neanderthals and the modern human colonization of Europe. Nature, 432, p. 461-465.

MELLARS P. 2006 - Archeology and the dispersal of modern humans in Europe : deconstructing the "Aurignacian". Evolutionary Anthropology, 15, p. 167-182.

MOGOSANU F. 1983 - L'Aurignacien du Banat. In : Aurignacien et Gravettien en Europe. Liège, ERAUL 13, p. 223-237.

MONS L. 1981 - L'industrie osseuse du gisement du Piage. In : F. Champagne et R. Espitalié (Dir.), Le Piage, site préhistorique du Lot. Paris, Société Préhistorique Française, p. 135-146, (Mémoires de la Société Préhistorique Française, 15).

NORMAND C. 2006 - L'Aurignacien de la salle de SaintMartin (Grotte d'Isturitz, commune de Saint-Martin d'Arberoue, Pyrénées Atlantiques) : données préliminaires sur l'industrie lithique recueillie lors des compagnes 20002002. In : F. Bon, J.M. Maillo Fernandez et D. Ortega i Cobos (Eds.), Autour des concepts de Protoaurignacien, d'Aurignacien archaïque, initial et ancien. Unité et variabili- 
té des comportements techniques des premiers groupes d'hommes modernes dans le Sud de la France et le Nord de l'Espagne. Madrid, UNED, p. 145-174, ( Serie I, Prehistoria y Arqueologia, 15).

NORMAND C., TURQ A. 2005 - L'Aurignacien de la grotte d'Isturitz (France) : la production lamellaire dans la séquence de la Salle de Saint-Martin. In : F. Le Brun-Ricalens (Ed.), Productions lamellaires attribuées à l'Aurignacien, chaînes opératoires et perspectives technoculturelles. Luxembourg, MNHA, p. 375-392, ( ArchéoLogiques, 1).

O'FARRELL M. 2005 - Etude préliminaire des éléments d'armature lithique de l'Aurignacien ancien de Brassempouy. In : F. Le Brun-Ricalens (Ed.), Productions lamellaires attribuées à l'Aurignacien, chaînes opératoires et perspectives technoculturelles. Luxembourg, MNHA, p. 395-412 ( ArchéoLogiques, 1).

ONORATINI G. 1986 - Découverte en Provence orientale (grotte Rainaude) d'une industrie souche de l'Aurignacien. Cette civilsation est-elle monolithique ? Bulletin de la Société Préhistorique Française, 83, p. 240-256.

ONORATINI G. 2004 - Le Protoaurignacien : première culture de l'homme moderne de Provence et Ligurie. L'Anthropologie, 108, p. 239-249.

ORSCHIEDT J. 2002 - Datation d'un vestige humain provenant de La Rochette (Saint-Léon-sur-Vézère, Dordogne) à partir de la méthode du carbone 14 en spectrométrie de masse. Paléo, 14, p. 239-240.

ORTEGA COBOS D., SOLER I MASFERRER N., MAROTO J. 2005 - La production de lamelles pendant l'Aurignacien archaïque dans la grotte de l'Arbreda (Espagne) : organisation de la production, variabilité des méthodes et objectifs. In : F. Le Brun-Ricalens (Ed.), Productions lamellaires attribuées à l'Aurignacien, chaînes opératoires et perspectives technoculturelles. Luxembourg, MNHA, p. 359-374, (ArchéoLogiques, 1).

OTTE M. 1990 - From the Middle to the Upper Paleolithic: the nature of the transition. In : P. Mellars (Ed.), The emergence of modern humans : an archaeological perspective. Edinburgh, Edinburgh University Press, p. 438-456.

OTTE M., KOZLOWSKI J.K. 2004 - La place du Baradostien dans l'origine du Paléolithique supérieur d'Eurasie. L'Anthropologie, 108, p. 395-406.

PAUNESCU A. 2001 - Paleoliticul si Mezoliticul din Spatiul Transilvan. Studiu monografic. Bucharest, Agir.

PELEGRIN J. 1995 - Technologie lithique : le Châtelperronien de Roc-de-Combe (Lot) et de La Côte (Dordogne). Paris, CNRS Editions.

PELEGRIN J., O'FARRELL M. 2005 - Les lamelles retouchées ou utilisées de Castanet. In : F. Le Brun-Ricalens (Ed.), Productions lamellaires attribuées à l'Aurignacien, chaînes opératoires et perspectives technoculturelles. Luxembourg, MNHA, p. 103-121, (ArchéoLogiques, 1).

PELEGRIN J., SORESSI M. 2007 - Le Châtelperronien et ses rapports avec le Moustérien. In : B. Vandermeersch et B. Maureille (Eds.), Les Néandertaliens : biologie et cultures. Paris, CTHS (Documents Préhistoriques, 33).

RICHTER D., WAIBLINGER J., RINK W.J., WAGNER G.A. 2000 - Thermoluminescence, Electronic Spin Resonance and ${ }^{14} \mathrm{C}$-dating of the Late Middle and Early Upper Palaeolithic site of Geissenklösterle cave in Southern Germany. Joumal of Archaeological Science, 27, p. 71-89.

RIGAUD J.-P. 1993 - L'Aurignacien dans le sud-ouest de la France : bilan et perspectives. In : L. Bánesz et J.K. Kozlowski (Eds.), Aurignacien en Europe et au ProcheOrient. Bratislava, Institut Archéologique de l'Académie Slovaque des Sciences, p. 181-186.

RIGAUD J.-P. 1996 - L'émergence du Paléolitique supérieur en Europe occidentale. Le rôle du Castelperronien. In : O. BarYoseph, L. Cavalli-Sforza, R. March et M. Piperno (Eds.), The Lower and Middle Palaeolithic. Forli, ABACO, p 219-223.

RIGAUD J.-P. 2001 - A propos de la contemporanéité du Castelperronien et de l'Aurignacien dans le nord-est de l'Aquitaine : une révision des données et ses implications. In : J. Zilhão, T. Aubry et A.F. Carvalho (Eds.), Les premiers hommes modernes de la Péninsule ibérique. Lisbonne, Instituto Poruguese de Arqueologia, p. 61-68, (Trabalhos de Arqueologia, 17).

RIGAUD J.-P., LUCAS G. 2006 - The first Aurignacian technocomplexes in Europe : a revision of the Bachokirian. In : O. Bar-Osef et J. Zilhão (Eds.), Towards a definition of the Aurignacian. Lisbonne, Instituto Poruguese de Arqueologia, p. 277-284, (Trabalhos de Arqueologia, 45). SHEA J.J. 2006 - The origins of lithic projectile point technology : evidence from Africa, the Levant and Europe. Journal of Archaeological Science, 33, p. 823-846.

SHENNAN S. 2001 - Demography and cultural innovation : a model and its implication for the emergence of modern human culture. Cambridge Archaeological Journal, 11, p. 5-16.

SKRDLA P. 1996 - The Bohunician reduction strategy. Quaternaria Nova, 6, p. 93-107.

SKRDLA P. 2003 - Comparison of Boker Tachtit and Stránská skála MP/UP transitional industries. Joumal of the Israel Prehistoric Society, 33, p. 37-73.

SLIMAK L. 2004 - Les dernières expressions du Moustérien entre Loire et Rhône. Thèse de doctorat, Université de Provence.

SONNEVILLE-BORDES D. de (1958) - Problèmes généraux du Paléolithique supérieur dans le Sud-Ouest de la France. L'Anthropologie, 62, p. 413-451. 
SONNEVILLE-BORDES D. de (1960) - Le Paléolithique supérieur en Périgord. Bordeaux, Delmas.

SONNEVILLE-BORDES D. de (1966) - L'évolution du Paléolithique supérieur en Europe occidentale et sa signification. Bulletin de la Société Préhistorique Française, LXIII, p. 3-34.

STRAUS L.G. 1996 - Continuity or rupture; convergence or invasion; adaptation or catastrophe; mosaic or monolith : views on the Middle to Upper Paleolithiic transition in Iberia. In : E. Carbonell et M. Vaquero (Eds.), The last Neandertals, the first Anatomically Modern Humans : a tale about the human diversity. Cultural change and human evolution : the crisis at $40 \mathrm{ka} \mathrm{BP}$. Tarragona, Universitat Rovira i Virgili, p. 203-218.

STRAUS L.G. 2003 - "The Aurignacian"? Some thoughts. In : J. Zilhão, F. d'Errico (Eds.), The chronology of the Aurignacian and of the transitional technocomplexes. Dating, stratigraphies, cultural implications. Lisbonne, Instituto Português de Arqueologia, p. 11-17, (Trabalhos de Arqueologia, 33).

STREET M., TERBERGER T., ORSCHIEDT J. 2006 - A critical review of the German Paleolithic hominin record. Journal of Human Evolution, 51, p. 551-579.

STROBL J., OBERMAIER H. 1909 - Die Aurignacienstation von Krems (Niederösterreich). Jahrbuch für Altertumskunde, 3, p. 129-148.

TABORIN Y. 1993 - La parure en coquillage au Paléolithique. Paris, CNRS Editions, (Supplément à Gallia Préhistoire, 29).

TARTAR E., TEYSSANDIER N., BON F., LIOLIOS D. 2006 - Equipement de chasse, équipement domestique : une distinction efficace ? Réflexion sur la notion d'investissement technique dans les industries aurignaciennes In : L. Astruc, F. Bon, V. Léa, P.-Y. Milcent et S. Phillibert (Eds.), Normes techniques et pratiques sociales : de la simplicité des outillages pré- et protohistoriques. Antibes, ADPCA, p. 107-118, (Actes des XXVlèmes Rencontres Internationales d'Archéologie et d'Histoire d'Antibes).

TEYSSANDIER N. 2003 - Les débuts de l'Aurignacien en Europe. Discussion à partir des sites de Geissenklösterle, Willendorf II, Krems-Hundssteig et Bacho Kiro. Thèse de doctorat, Université Paris X-Nanterre.

TEYSSANDIER N. 2006 - Questioning the first Aurignacian: mono or multi cultural phenomenon during the formation of the Upper Paleolithic in Central Europe and the Balkans. Anthropologie, International Journal of the Science of Man, XLIV, 1, p. 9-29.

TEYSSANDIER N. 2007 - En route vers l'Ouest. Les débuts de l'Aurignacien en Europe. Oxford, John and Erica Hedges Ltd., (BAR International Series, 1638).
TEYSSANDIER N., LIOLIOS D. 2003 - Defining the earliest Aurignacian in the Swabian Alp : the relevance of the technological study of the Geissenklösterle (Blaubeuren, Germany) lithic and organic productions. In : J. Zilhão et F. d'Emico (Eds.), The chronology of the Aurignacian and of the transitional technocomplexes. Dating, stratigraphies, cultural implications. Lisbonne, Instituto Portuguese de Arqueologia, p. 179-196, (Trabalhos de Arqueologia, 33).

TEYSSANDIER N., BOLUS M., CONARD N.J. 2006 - The first Aurignacian in Central Europe and its place in an European perspective. In : O. Bar-Yosef et J. Zilhão (Eds.), Towards a definition of the Aurignacian. Lisbonne, Instituto Portuguese de Arqueologia, p. 241-256, (Trabalhos de Arqueologia, 45).

TEYSSANDIER N., MESTER Z., LENGYEL G. en préparation - Istállóskö revisited. Technological and taphonomic analyses of a key-sequence for the Middle-to-Upper Paleolithic transition in central Europe.

TOSTEVIN G. 2000 - The Middle to Upper Paleolithic transition from the Levant to Central Europe : in situ development or diffusion ? In : J. Orschiedt et C.-G. Weniger (Eds.), Neanderthals and Modern Humans - Discussing the transition : Central and Eastern Europe from 50 000-30 000 B.P. Mettmann, Neanderthal Museum, p. 92-112.

TRINKAUS E. 2005 - Early modern humans. Annual Review of Anthropology, 34, p. 207-230.

TSANOVA T. 2006 - Les débuts du Paléolithique supérieur dans l'Est des Balkans. Réflexion à partir de l'étude taphonomique et techno-économique des ensembles lithiques des sites de Bacho Kiro (couche 11), Temnata (couches VI et 4) et Kozarnika (couche VII). Thèse de doctorat, Université Bordeaux 1.

TSANOVA T., BORDES J.-G. 2003 - Contribution au débat sur l'origine de l'Aurignacien : principaux résultats d'une étude technologique de l'industrie lithique de la couche 11 de Bacho Kiro. In : T. Tsonev et E. Montagnari Kokelj (Eds.) : The humanized mineral world : towards social and symbolic evaluation of prehistoric technologies in South Eastem Europe. Liège, ERAUL, 103, p. 41-50.

VALOCH K. 1990 - La Moravie il y a 40000 ans. In : C. Farizy (Ed.), Paléolithique moyen récent et Paléolithique supérieur ancien en Europe. Nemours, APRAIF, p. 115-124, (Mémoires du Musée de Préhistoire d'lle-de-France, 3).

VALLADAS H., TISNÉRAT-LABORDE N., CACHIER H., KALTNECKER E., ARNOLD M., OBERLIN C., EVIN J. 2005 Bilan des datations carbone 14 effectuées sur des charbons de bois de la grotte Chauvet. Bulletin de la Société Préhistorique Française, 102, 1, p. 109-113.

VANHAEREN M. 2002 - Les fonctions de la parure au Paléolithique supérieur : de l'individu à l'unité culturelle. Thèse de doctorat, Université Bordeaux 1. 
VANHAEREN M., D'ERRICO F. 2006 - Aurignacian ethnolinguistic geography of Europe revealed by personal orn aments. Journal of Archaeological Science, 33, p. 11051128.

VERPOORTE A. 2005 - The first modern humans in Europe ? A closer look at the dating evidence from the Swabian Jura (Germany). Antiquity, 79, p. 269-279.

WHITE R. 1989 - Production complexity and standardization in early Aurignacian bead and pendant manufacture. In : P. Mellars et C. Stringer (Eds.), The human revolution : behavioural and biological perspectives on the origins of modem humans. Princeton, Princeton University Press, p. 366-390.

WILD E.M., Teschler-Nicola M., Kutschera W., Steier P., Trinkaus E., Wanek W. 2005 - Direct dating of Early Upper Palaeolithic human remains from Mlade_. Nature, 435, p. 332-335.

ZILHÃO J. 2006 - Neandertals and Moderns mixed, and it matters. Evolutionary Anthropology, 15, p. 183-195.
ZILHÃO J. 2007 - The emergence of ornaments and art : an archaeological perspective on the origins of " behavioral modemity ". Jou mal of Archaeological Researc $h, 15, \mathrm{p}$. $1-54$.

ZILHÃO J., D'ERRICO F. 1999 - The chronology and taphonomy of the Earliest Aurignacian and Its implications for the understanding of Neandertal extinction. Journal of World Prehistory, 13, 1, p. 1-68.

ZILHÃO J., D'ERRICO F. 2003 a - The chronology of the Aurignacian and of the Transitional technocomplexes. Where do we stand ? In : J. Zilhão et F. d'Errico (Eds.), The chronology of the Aurignacian and of the transitional technocomplexes. Dating, stratigraphies, cultural implications. Lisbonne, Instituto Portuguese de Arqueologia, p. 313349, (Trabalhos de Arqueologia, 33).

ZILHÃO J., D'ERRICO F. 2003 b - An Aurignacian "garden of Eden" in southern Germany ? An alternative interpretation of the Geissenklösterle and a critique of the Kulturpumpe model. Paléo, 15, p. 69-86. 
